\title{
A Bayesian Approach for Localization of Acoustic Emission Source in Plate-Like Structures
}

\author{
Gang Yan and Jianfei Tang \\ State Key Laboratory of Mechanics and Control of Mechanical Structures, Nanjing University of Aeronautics and Astronautics, \\ Nanjing 210016, China \\ Correspondence should be addressed to Gang Yan; yangang@nuaa.edu.cn
}

Received 26 August 2014; Revised 15 December 2014; Accepted 15 December 2014

Academic Editor: Igor Andrianov

Copyright (C) 2015 G. Yan and J. Tang. This is an open access article distributed under the Creative Commons Attribution License, which permits unrestricted use, distribution, and reproduction in any medium, provided the original work is properly cited.

\begin{abstract}
This paper presents a Bayesian approach for localizing acoustic emission (AE) source in plate-like structures with consideration of uncertainties from modeling error and measurement noise. A PZT sensor network is deployed to monitor and acquire AE wave signals released by possible damage. By using continuous wavelet transform (CWT), the time-of-flight (TOF) information of the AE wave signals is extracted and measured. With a theoretical TOF model, a Bayesian parameter identification procedure is developed to obtain the AE source location and the wave velocity at a specific frequency simultaneously and meanwhile quantify their uncertainties. It is based on Bayes' theorem that the posterior distributions of the parameters about the AE source location and the wave velocity are obtained by relating their priors and the likelihood of the measured time difference data. A Markov chain Monte Carlo (MCMC) algorithm is employed to draw samples to approximate the posteriors. Also, a data fusion scheme is performed to fuse results identified at multiple frequencies to increase accuracy and reduce uncertainty of the final localization results. Experimental studies on a stiffened aluminum panel with simulated AE events by pensile lead breaks (PLBs) are conducted to validate the proposed Bayesian AE source localization approach.
\end{abstract}

\section{Introduction}

The increasing emphasis on integrity of critical structures such as aircrafts urges the needs to monitor structures in situ and real-time to detect damages at an early stage to prevent catastrophic failure. In general, an effective online structural health monitoring system should consist of two major components: active monitoring and passive monitoring [1, 2]. Active monitoring needs both actuators and sensors to evaluate the damage of a structure, while passive monitoring uses only sensors to "listen" to the acoustic emission (AE) wave signals emitted by internal or external sources such as propagating cracks or impacts. In the present study, we deal with passive monitoring to determine the $\mathrm{AE}$ source location in plate-like structures where possible damage may exist.

Up to date, a lot of AE source localization approaches have been developed, including several relatively new introduced ones, such as genetic algorithms [3], neural networks [4], support vector machines [5], time reversal [6], beamforming [7], and Voronoi construction [8]. Among these approaches, the triangulation-based approaches utilizing the time-of-flight (TOF) information of the $\mathrm{AE}$ wave signals are most widely used [9-14]. With the known positions of the sensors and the velocities of the $\mathrm{AE}$ wave signals, the location of the AE source can be determined by solving a set of nonlinear equations directly or by iterative optimization algorithms. These triangulation approaches are originally developed for isotropic structures and then extended to anisotropic composite structures, for which damage caused by low velocity impact is a major concern $[13,14]$. In these approaches, the time arrivals are usually obtained by threshold crossing [15], cross-correlation [16], and statistical Akaike information criterion (AIC) [17]. With the development of modal acoustic emission (MAE) technique in which the concept of guided waves is introduced to improve the interpretation of the $\mathrm{AE}$ wave signals, the continuous wavelet transform (CWT) has become a useful tool for the time-frequency representation of transient $\mathrm{AE}$ waves in dispersive medium with its advantage of good resolution in both time and frequency domains. It makes the determination of the time arrivals of the dispersive $\mathrm{AE}$ waves at each local frequency more accurate $[10,11,18-$ 20]. Other advanced signal processing techniques, such as 
Hilbert transform (HT) [21], short time Fourier transform (STFT) [22], and warped Fourier transform (WFT) [23] have been also employed for processing the AE wave signals in recent years.

Generally, most of the triangulation approaches developed can be categorized as deterministic methods, in which uncertainties are not considered and only single solution is given as the final result. However, in reality errors and uncertainties are always unavoidable in the measurement and identification process. For example, the time arrival determined by threshold crossing is usually vulnerable to the measurement noise. Even by employing CWT, systematic uncertainty could exist due to the Heisenberg uncertainty principle [20]. In addition, uncertainties may arise from the theoretical wave velocities, since they depend on the material property values of a specific structure, which may have variations from those nominal values and be influenced by several factors, such as environmental temperature changes [24]. Under such a circumstance, probabilistic approaches may be more appropriate than deterministic approaches for AE source localization in that probability distributions can be used to quantify the various uncertainties. This leads to the research of probability-based approaches, to give a more reasonable source characterization. Niri and Salamone proposed a probabilistic approach for AE source localization in plate-like structures based on extended Kalman filter (EKF). The main advantage of EKF algorithm over traditional methods is that it can take into account uncertainties in TOF measurement and wave velocity [20]. They also proposed an adaptive multisensor fusion framework to combine with EKF to locate the AE sources in noisy operation conditions [21]. Schumacher et al. proposed a Bayesian analysis approach for passive $\mathrm{AE}$ source location in concrete bridge column to account for uncertainties and errors that exist within the measurement and calculation process [25].

The aim of this work is to develop a Bayesian approach for localizing AE source in plate-like structures, while considering uncertainties from modeling error and measurement noise. It is an extension of an early work dealing with active monitoring of damage for plate-like structures using Lamb waves [26]. The rest of this paper is structured as follows. Section 2 describes the proposed Bayesian approach for $\mathrm{AE}$ source localization and outlines the main idea of Markov chain Monte Carlo (MCMC) method for sampling the posterior distributions of the unknown parameters, while a data fusion scheme to increase accuracy and reduce uncertainty of the localization results is also presented. Experimental studies on a stiffened aluminum panel with simulated AE events are conducted in Section 3 to validate the proposed probabilistic localization approach. Final conclusions are given in Section 4.

\section{Bayesian Approach for AE Source Localization}

2.1. TOF-Based AE Source Localization. For plate-like structures, a sensor network consisting of $N_{s}$ sensors is deployed to passively monitor the AE wave signals released by potential damage. Among these $N_{s}$ sensors, one is selected as a trigger

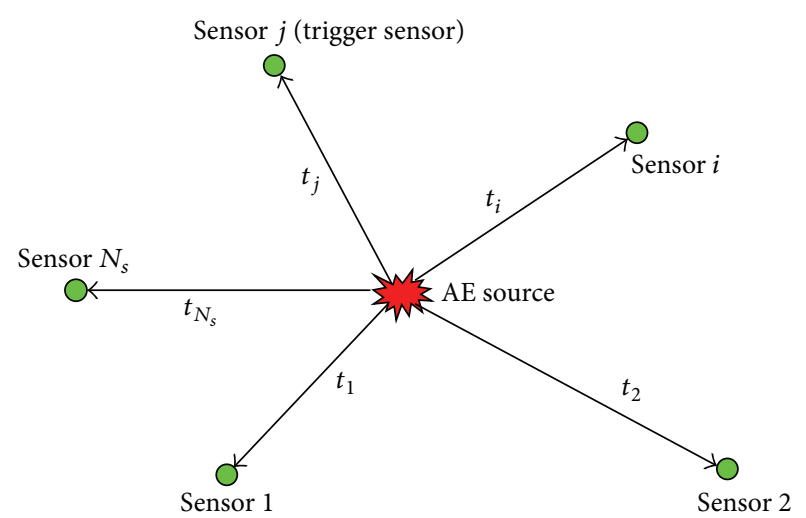

FIGURE 1: Illustration of AE source localization and sensor network.

sensor (or master sensor). Assume that the sensor network works in such a mode: once the voltage level of the data acquisition (DAQ) channel connected to the trigger sensor exceeds a predefined threshold value (usually higher than the noise level), an AE event is regarded to have occurred; all of the channels of the sensor network start to acquire the $\mathrm{AE}$ wave signals simultaneously and save them to the computer for further analysis. As illustrated in Figure 1, using the TOF of the $\mathrm{AE}$ waves is a straightforward way to triangulate the $\mathrm{AE}$ source; however, the absolute time of the initiation of an $\mathrm{AE}$ event is usually unknown, only the time differences between the $\mathrm{AE}$ wave signals received by the rest of the sensors and that received by the trigger sensor can be measured after the time arrivals in each channel are extracted. Theoretically, the time difference between the $i$ th sensor and the trigger sensor (assume it is the $j$ th sensor) $\Delta t_{i j}^{c}$ can be calculated as

$\Delta t_{i j}^{c}=\frac{\sqrt{\left(x_{d}-x_{i}\right)^{2}+\left(y_{d}-y_{i}\right)^{2}}-\sqrt{\left(x_{d}-x_{j}\right)^{2}+\left(y_{d}-y_{j}\right)^{2}}}{V_{g}}$,

where $\left(x_{d}, y_{d}\right),\left(x_{i}, y_{i}\right)$, and $\left(x_{j}, y_{j}\right)$ are the coordinates of the AE source location and the $i$ th and $j$ th sensors, respectively. $V_{g}$ is the wave velocity of the AE waves at a specific frequency. Usually, in plate-like structures, the AE waves contain symmetric and antisymmetric modes of guided waves governed by Rayleigh-Lamb equation [27], and $V_{g}$ is dependent of the selected frequency due to the dispersive nature of guide waves, resulting in $\Delta t_{i j}^{c}$ becoming dependent of frequency as well. In the present study, only isotropic plate is considered; $V_{g}$ is independent of propagation direction. Since uncertainties from material properties could influence the accuracy of the theoretical wave velocity value, in the present study, the wave velocity $V_{g}$ in (1) is set as an additional unknown parameter to be identified besides the coordinates of the $\mathrm{AE}$ source location, rather than calculated using the nominal material property values beforehand by solving RayleighLamb equation or by using approximate plate theory solution [28]. Thus, the unknown parameters that should be identified in our AE source localization approach are the center location of the AE source $\left(x_{d}, y_{d}\right)$ and the wave velocity $V_{g}$ at 
a specific frequency. They can be written in a vector style as $\boldsymbol{\theta}=\left[x_{d}, y_{d}, V_{g}\right]^{T}$.

For localizing the AE source using TOF-based method, the time arrivals of the AE wave signals in each channel at specific frequencies should be obtained first. As aforementioned in the introduction, CWT has good resolution in both time and frequency domains; it is popular in analyzing dispersive guided waves. In the present study, a complex Morlet wavelet is employed to determine the time arrivals of the $\mathrm{AE}$ wave signals in the interested frequencies. The details of CWT of complex Morlet wavelet can be found in [11, 20] and are not repeated here for conciseness. Theoretical and experimental results have demonstrated that the first peak of the magnitude of CWT coefficients represents the arrival time of the dispersive wave with the group velocity at local frequencies $[10,11,18-20]$. This important property is used in the present study to extract the time arrivals of the AE waves in each channel. After that, the measured time difference between the $i$ th sensor and the trigger sensor $\Delta t_{i j}^{m}$ can be measured simply as

$$
\Delta t_{i j}^{m}=t_{i}^{m}-t_{j}^{m}
$$

in which $t_{i}^{m}$ and $t_{j}^{m}$ are the measured time arrivals of the $i$ th sensor and the trigger sensor (the $j$ th sensor), respectively.

2.2. Bayesian Approach for Parameter Identification. After the unknown parameters are defined, the AE source localization is formulated as a parameter identification problem with the measured time difference data. In the present study, it is solved from a probabilistic point of view by employing Bayesian identification. Rather than pinpointing a single solution by deterministic approaches, the Bayesian approach can provide probabilistic distributions of the unknown parameters, giving both point and interval estimates [2934]. The basic idea of Bayesian identification is that it treats the parameters, currently denoted by a vector $\boldsymbol{\theta}$, as random variables with joint distribution $p(\boldsymbol{\theta})$. It aims to update the posterior distributions of the uncertain parameters for a given set of measured data. The final parameter estimates can be taken as the mean values of the posteriors or use the values that maximize the posteriors.

Assume that the uncertainties from modeling error and measurement noise can be described by a Gaussian type random variable $\varepsilon$; the probabilistic description of the measured time difference between the $i$ th sensor and the triggersensor can be expressed as

$$
\Delta t_{i j}^{m}=\Delta t_{i j}^{c}(\boldsymbol{\theta})+\varepsilon
$$

where $\Delta t_{i j}^{c}$ is the theoretically calculated time difference for the $i$ th sensor using (1), with the coordinates of AE source location and the wave velocity contained in the parameter vector $\boldsymbol{\theta}, \boldsymbol{\varepsilon}$ is usually assumed as a variable with mean of zero and variance of $\sigma_{\varepsilon}^{2}$, and assumption is made that the uncertainties from different sensors have the same variance and they are uncorrelated. In this case, the likelihood function can be written as

$$
p(\mathbf{D} \mid \boldsymbol{\theta})=\frac{1}{\left(2 \pi \sigma_{\varepsilon}^{2}\right)^{N_{s} / 2}} \exp \left[-\frac{1}{2 \sigma_{\varepsilon}^{2}} \sum_{i=1}^{N_{s}}\left(\Delta t_{i j}^{m}-\Delta t_{i j}^{c}(\boldsymbol{\theta})\right)^{2}\right] .
$$

The likelihood function $p(\mathbf{D} \mid \boldsymbol{\theta})$ is a probabilistic statement about the distribution of the measured time difference data $\mathbf{D}=\left[\begin{array}{llll}\Delta t_{1 j}^{m} & \Delta t_{2 j}^{m} & \cdots & \Delta t_{N_{s}}^{m}\end{array}\right]^{T}$ given the predicted time difference values, determined by (1) and parameter vector $\boldsymbol{\theta}$. Usually, the variance $\sigma_{\varepsilon}^{2}$ is unknown, and a conjugate Gamma prior with parameters $a$ and $b$ is adopted for the precision $\tau=1 / \sigma_{\varepsilon}^{2}$ in the present study [24]. With this choice, the variance can be analytically integrated out from (4), leading to a new likelihood expression as

$$
\begin{aligned}
p(\mathbf{D} \mid \boldsymbol{\theta})= & \frac{\Gamma\left(a+N_{s} / 2\right) b^{a}}{\Gamma(a)(2 \pi)^{N_{s} / 2}} \\
& \times\left[b+\frac{1}{2} \sum_{i=1}^{N_{s}}\left(\Delta t_{i j}^{m}-\Delta t_{i j}^{c}(\boldsymbol{\theta})\right)^{2}\right]^{-\left(a+N_{s} / 2\right)},
\end{aligned}
$$

where $\Gamma(z)=\int_{0}^{+\infty} t^{z-1} e^{-t} d t$ is the Gamma function. This new likelihood function instead of (4) is used in the following calculation.

By using Bayes' theorem, the posterior distribution of parameter vector $\boldsymbol{\theta}$ for a given set of measured time difference data $\mathbf{D}$ is constructed by relating the prior and the likelihood function as

$$
p(\boldsymbol{\theta} \mid \mathbf{D})=\frac{p(\mathbf{D} \mid \boldsymbol{\theta}) p_{\pi}(\boldsymbol{\theta})}{p(\mathbf{D})}
$$

where $p(\boldsymbol{\theta} \mid \mathrm{D})$ is the posterior distribution of $\boldsymbol{\theta}, p_{\pi}(\boldsymbol{\theta})$ is the prior distribution of $\boldsymbol{\theta}$, and $p(\mathbf{D})$ is a normalizing constant that ensure the integration of the distribution over the predefined domain is equal to unity. For each parameter $\theta_{k}(k=1,2,3)$, the marginal posterior distribution can be obtained by integrating (6) with respect to the rest parameters over the domain of interest as

$$
p\left(\theta_{k} \mid \mathbf{D}\right)=\int p(\boldsymbol{\theta} \mid \mathbf{D}) d \boldsymbol{\theta}_{-k} \propto \int p(\mathbf{D} \mid \boldsymbol{\theta}) p(\boldsymbol{\theta}) d \boldsymbol{\theta}_{-k},
$$

where the notation $\int d \boldsymbol{\theta}_{-k}$ denotes the multidimensional integral over all of the rest parameters other than $\theta_{k}$ in $\boldsymbol{\theta}$.

2.3. Markov Chain Monte Carlo Method. Equation (7) gives a general expression of the updated marginal distribution for each parameter in $\boldsymbol{\theta}$ using measured time difference data. After that, the parameters can be estimated from the posterior distributions, and the AE source location and the wave velocity can be identified. However, analytical evaluation of (7) is usually difficult since it involves integration over multidimensional parameters. In the present study, MCMC method is resorted to obtain samples to approximate the posterior distributions and make inference. 


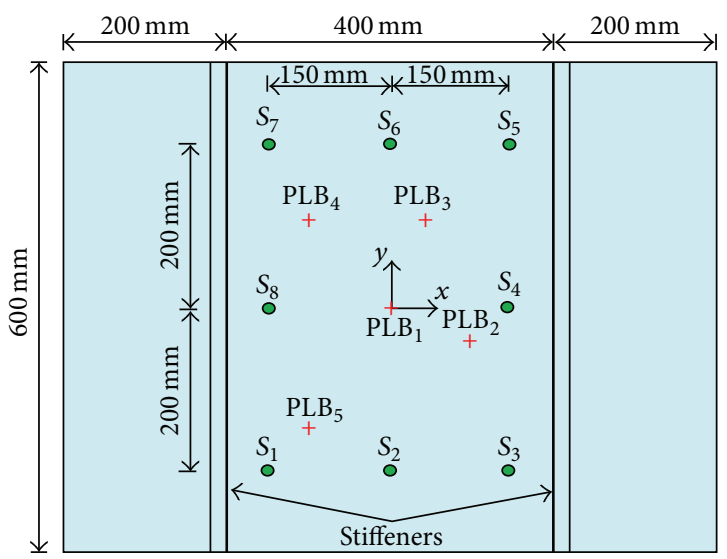

Figure 2: Test configuration and sensor arrangement for the stiffened aluminum panel.

The pioneer work of MCMC was done by Metropolis and his colleagues in the 1950s. They recognized that Markov chains could be applied to some difficult sampling problems. After about two decades, Hastings expanded and formalized MCMC into the procedure now known as MetropolisHastings $(\mathrm{M}-\mathrm{H})$ algorithm for performing such sampling [35]. Let $\pi(\theta)$ be the target distribution from which random samples are desired. The $\mathrm{M}-\mathrm{H}$ algorithm generates a sequence of $\boldsymbol{\theta}^{l}$ for the target distribution $\pi(\boldsymbol{\theta})$ by a two-step rejection sampling procedure. At stage $l$, a candidate value $\boldsymbol{\theta}^{*}$ is sampled based on the current value $\boldsymbol{\theta}^{l-1}$; it is sampled from a chosen proposal or transition distribution function $h\left(\boldsymbol{\theta}^{*} \mid \boldsymbol{\theta}^{l-1}\right)$. A Bernoulli trial is performed with success probability

$$
\alpha_{\text {accept }}=\min \left\{\frac{\pi\left(\boldsymbol{\theta}^{*}\right) h\left(\boldsymbol{\theta}^{l-1} \mid \boldsymbol{\theta}^{*}\right)}{\pi\left(\boldsymbol{\theta}^{l-1}\right) h\left(\boldsymbol{\theta}^{*} \mid \boldsymbol{\theta}^{l-1}\right)}, 1\right\} .
$$

If the result of the trial is success, $\boldsymbol{\theta}^{l}$ is set to $\boldsymbol{\theta}^{*}$; otherwise $\boldsymbol{\theta}^{l}$ is kept to $\boldsymbol{\theta}^{*}$. This process is repeated for a sufficient number of iterations, until the resulting Markov chain is stationary and can be used to represent the target distribution. Usually, when starting from an arbitrary state, there is a nonstationary period of iterations before the chain gets converged and stationary. It is called "burn-in" period and should be discarded in the final approximation of the posterior distribution. The proposal distribution could be an arbitrary distribution specified by the user, but proper ones can lead to better convergence in the resulting Markov chain. Often symmetrical proposal distribution, which means $h\left(\boldsymbol{\theta}^{*}\right.$ | $\left.\boldsymbol{\theta}^{l-1}\right)$ is equal to $h\left(\boldsymbol{\theta}^{l-1} \mid \boldsymbol{\theta}^{*}\right)$, is used to simplify the acceptance probability.

In the present study, a modified $\mathrm{M}-\mathrm{H}$ algorithm is employed to sample the posterior distributions of the unknown parameters in $\boldsymbol{\theta}$ defined in Section 2.1. It is similar to the algorithm developed in $[26,33]$. This algorithm combines Gibbs sampling concept that the full conditional distribution of each parameter can be thought of as its posterior distribution if other parameters' values are known.
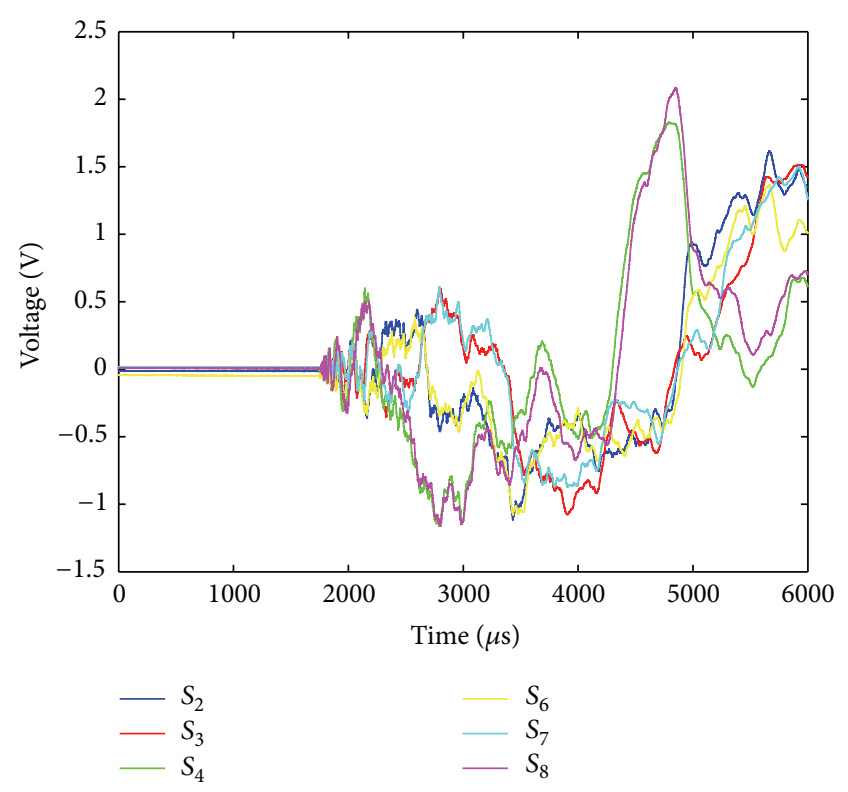

FIGURE 3: AE wave signals for simulated AE event $\mathrm{PLB}_{1}$.

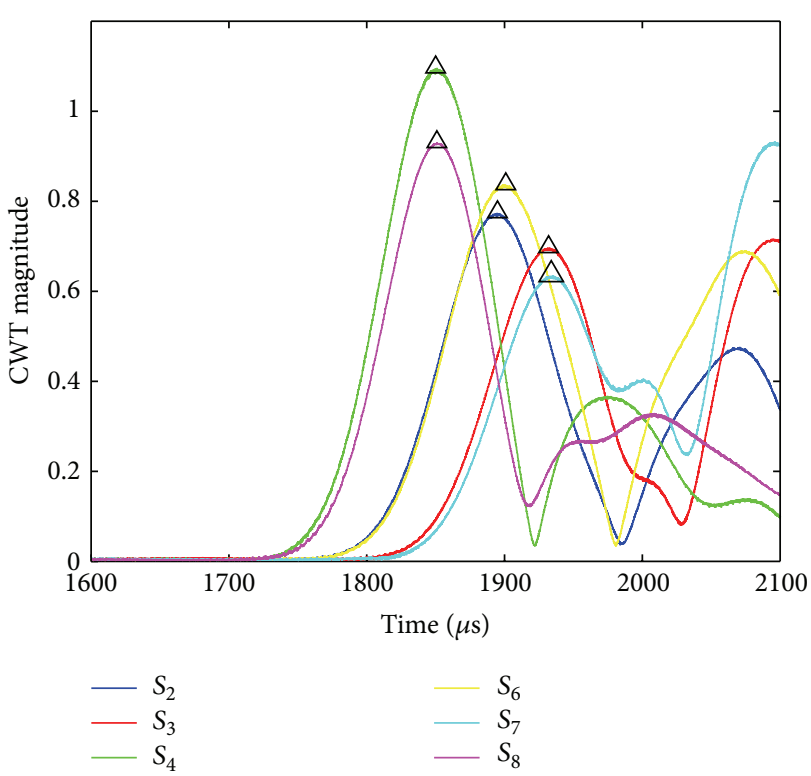

FIGURE 4: CWT of AE wave signals at $20 \mathrm{kHz}$ for simulated AE event $\mathrm{PLB}_{1}$.

It updates each parameter sequentially by using the most recent sampled values. Uniform transition distribution is employed to propose candidate value via a random walk scheme. For each parameter, the interval of random walk has been continuously tuned during the "burn-in" period to achieve an appropriate acceptance rate and improve the performance of MCMC with scaling factors suggested in [33]. Unlike the algorithm in [33], since the variance is already integrated out from the likelihood function, sampling of the parameters is independent of the variance; it is not necessary to sample the variance within the algorithm. 


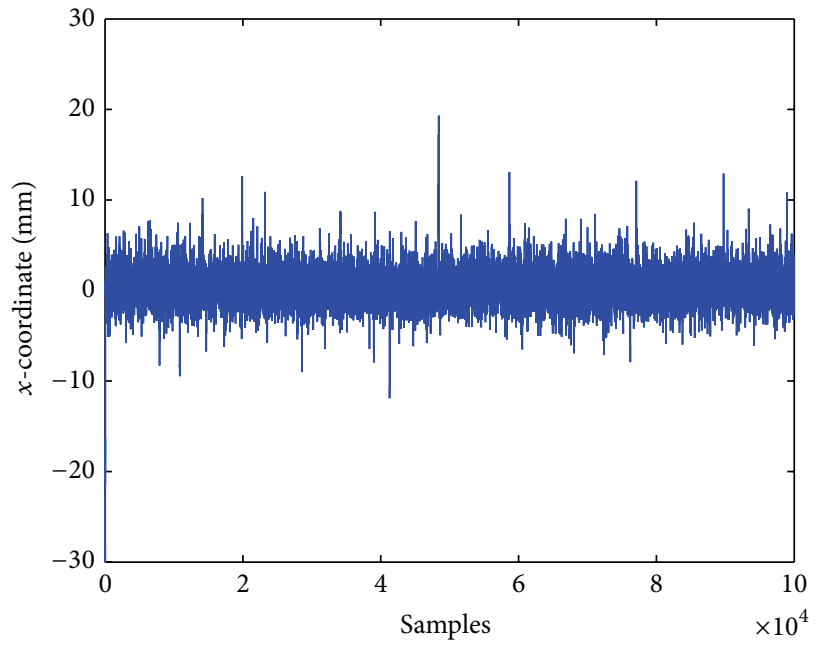

(a)

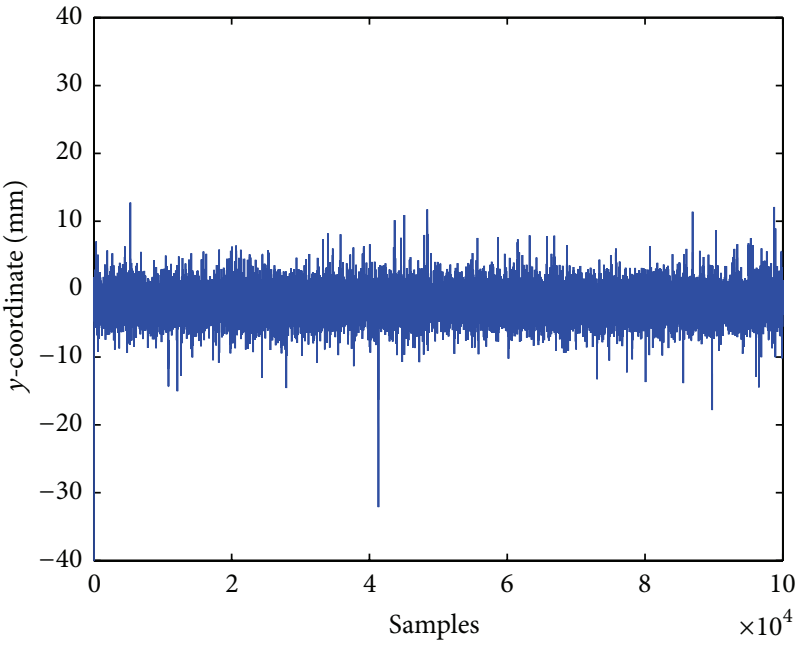

(b)

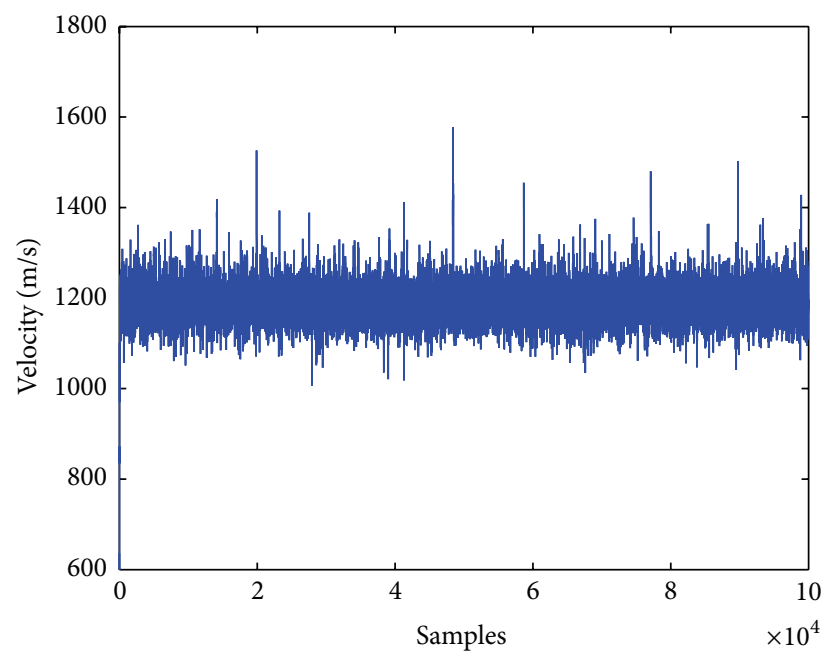

(c)

FIGURE 5: MCMC samples for simulated AE event PLB 1 (a) $x$-coordinate, (b) $y$-coordinate, and (c) wave velocity at $20 \mathrm{kHz}$.

2.4. Data Fusion at Multiple Frequencies. One of the advantages of extracting TOF information by CWT in timefrequency domain over other traditional methods is that we can obtain TOF data at multiple frequencies. All of these data at different frequencies can be used to localize the AE source individually with the proposed Bayesian approach. A proper data fusion scheme can be employed to increase accuracy and reduce uncertainty of the AE source locations. As suggested by $\mathrm{Su}$ et al. [36], usually three fusion architectures, that is, independent, centralized, and decentralized, can be chosen to perform data fusion. In the present study, an independent architecture is applied to fuse the results; namely, individually obtained AE source locations by time difference data at multiple frequencies are fused to get the final results.

After the MCMC algorithm obtains samples from the posterior distributions, it is reasonable to use Gaussian distribution to approximate these posteriors. For plate-like structures, an AE source location can be represented by a two-dimensional Gaussian distribution in a canonical form as

$$
p(X)=\frac{1}{2 \pi|\Sigma|^{1 / 2}} \exp \left[-\frac{1}{2}(X-\bar{X})^{T} \Sigma^{-1}(X-\bar{X})\right],
$$

where $\bar{X}=\left[\begin{array}{ll}\bar{x}_{d} & \bar{y}_{d}\end{array}\right]^{T}$ and $\Sigma=\left[\begin{array}{cc}\sigma_{x}^{2} & \rho \sigma_{x} \sigma_{y} \\ \rho \sigma_{x} \sigma_{y} & \sigma_{y}^{2}\end{array}\right]$ are the mean and covariance matrix of the distribution, respectively; $\bar{x}_{d}$ and $\bar{y}_{d}$ are means of the $x$-and $y$-coordinates of the identified AE source location; $\sigma_{x}$ and $\sigma_{y}$ are standard deviations of the $x$ - and $y$-coordinates; $\rho$ is the correlation coefficient, respectively.

Since individual localization results with Gaussian distributions can be obtained by time difference data at multiple frequencies, a fusion method is required to determine the combined parameters from those of individual distributions. In the present study, formulations for merging Gaussian distributions in $[37,38]$ are adopted. For Gaussian distributions, the mean, standard deviations, and orientation 
TABLE 1: Coordinates of the sensors on the stiffened aluminum panel.

\begin{tabular}{ccccccc}
\hline & $S_{2}$ & $S_{3}$ & $S_{4}$ & $S_{6}$ & $S_{7}$ & $S_{8}$ \\
\hline Coordinates $(\mathrm{mm})$ & $(0,-200)$ & $(150,-200)$ & $(150,0)$ & $(0,200)$ & $(-150,200)$ & $(-150,0)$ \\
\hline
\end{tabular}

TABLE 2: Coordinates of the pensile lead breaks on the stiffened aluminum panel.

\begin{tabular}{lccccc}
\hline & $\mathrm{PLB}_{1}$ & $\mathrm{PLB}_{2}$ & $\mathrm{PLB}_{3}$ & PLB $_{4}$ & PLB $_{5}$ \\
\hline Coordinates $(\mathrm{mm})$ & $(0,0)$ & $(100,-50)$ & $(50,100)$ & $(-100,100)$ & $(-100,-150)$ \\
\hline
\end{tabular}

of the major axis are independent of scaling; they can be extracted from the resulting merged covariance matrices without considering absolute probability values [38]. Take two Gaussian distributions as an example; the covariance matrix of the resulting merged distribution can be combined by individual covariance matrices as

$$
\Sigma^{*}=\Sigma_{1}-\Sigma_{1}\left(\Sigma_{1}+\Sigma_{2}\right)^{-1} \Sigma_{1}
$$

where $\Sigma^{*}$ is the covariance matrix of the merged distribution and $\Sigma_{1}$ and $\Sigma_{2}$ are the covariance matrices of the two individual distributions. The mean of the resulting merged distribution is computed from the means and covariance matrices of the individual distributions as

$$
\bar{X}^{*}=\bar{X}_{1}-\Sigma_{1}\left(\Sigma_{1}+\Sigma_{2}\right)^{-1}\left(\bar{X}_{1}+\bar{X}_{2}\right),
$$

where $\bar{X}^{*}$ is the mean of the merged distribution and $\bar{X}_{1}$ and $\bar{X}_{2}$ are the means of the two individual distributions. More Gaussian distributions can be merged one by one with the above formulations.

\section{Experimental Study}

3.1. Experimental Set-Up. To demonstrate the effectiveness of the proposed Bayesian AE source localization method, experimental studies for a stiffened aluminum panel are conducted. The test set-up consists of a stiffened aluminum panel and a National Instrument PXI monitoring system incorporated with a PXI-5105 8-channel digitizer. The dimensions of the stiffened aluminum panel are $800 \mathrm{~mm} \times 600 \mathrm{~mm} \times 2 \mathrm{~mm}$. A sensor network containing eight PZT transducers, denoted by $S_{1}$ to $S_{8}$, with diameter of $10 \mathrm{~mm}$ and thickness of $1 \mathrm{~mm}$ are surface mounted on an area of $300 \mathrm{~mm} \times 400 \mathrm{~mm}$ between two riveted stiffeners. These sensors are connected to the DAQ channels of the digitizer for monitoring the AE event and collecting AE wave signals. Unfortunately, $S_{1}$ and $S_{5}$ malfunction in the following test; only the remaining six sensors are employed for AE source localization. In the present study, six sensors are sufficient to collect time difference data to identify the three unknown parameters; however, a later study on the fail-safe distribution of sensors could help to increase the reliability of the monitoring system [39]. An illustration of the stiffened panel and sensor placement is shown in Figure 2. The origin of the coordinate system is set at the center of the panel, and the coordinates of the functioning sensors are listed in Table 1. In the experiments, pensile lead breaks (PLBs) are used to simulate sources of

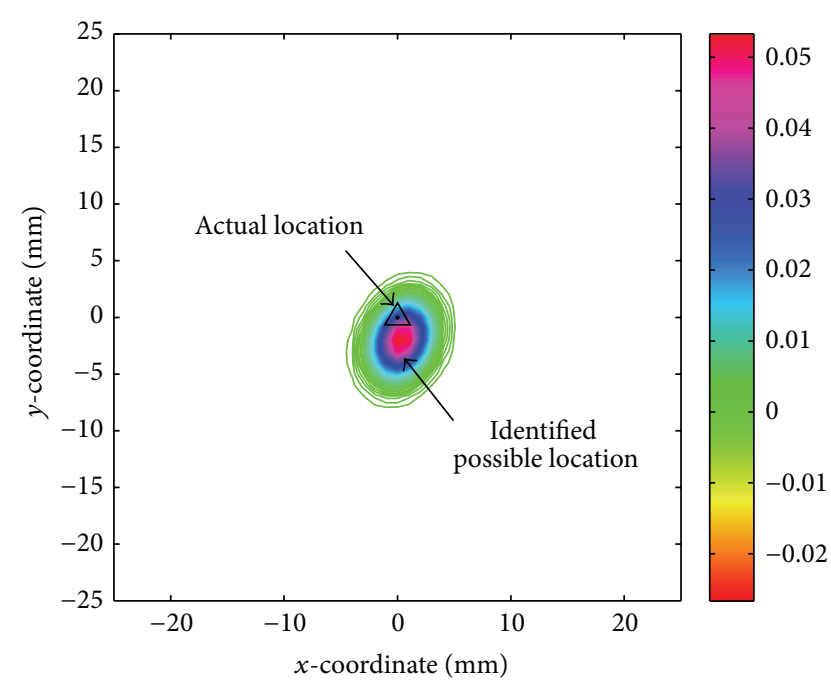

FIGURE 6: Identified source location for simulated AE event PLB $B_{1}$ by time difference data at $20 \mathrm{kHz}$.

AE events. Five PLBs at different locations are performed. These simulated AE sources are labeled in Figure 2 and their coordinates are listed in Table 2. During the experiments, $S_{4}$ is selected as the trigger sensor for the sensor network, and the threshold voltage value for triggering $S_{4}$ is set as $0.1 \mathrm{~V}$. After $S_{4}$ is triggered, the response wave signals are received by the sensors and acquired by the digitizer, whose sampling rate is set at $10 \mathrm{MHz}$.

\subsection{Experimental Results}

3.2.1. Results for Single-Frequency Data. Figure 3 shows the $A E$ wave signals emitted by the simulated $A E$ event $\mathrm{PLB}_{1}$ and received by the six sensors. To obtain the time arrivals of these AE wave signals, CWT of complex Morlet wavelet is applied. Figure 4 shows the magnitudes of CWT coefficients of the AE wave signals at frequency of $20 \mathrm{kHz}$. From the figure, the time arrivals of the AE wave signals represented by the first peaks can be clearly identified. The corresponding time arrivals for the AE wave signals at $20 \mathrm{kHz}$ are determined and labeled by triangles in Figure 4. Then the time difference data between the rest five sensors and the trigger sensor $S_{4}$ can be easily calculated by subtraction as listed in Table 3 .

After the time difference data is obtained, MCMC procedure is then performed to obtain samples for the unknown parameters to approximate their posterior distributions. 

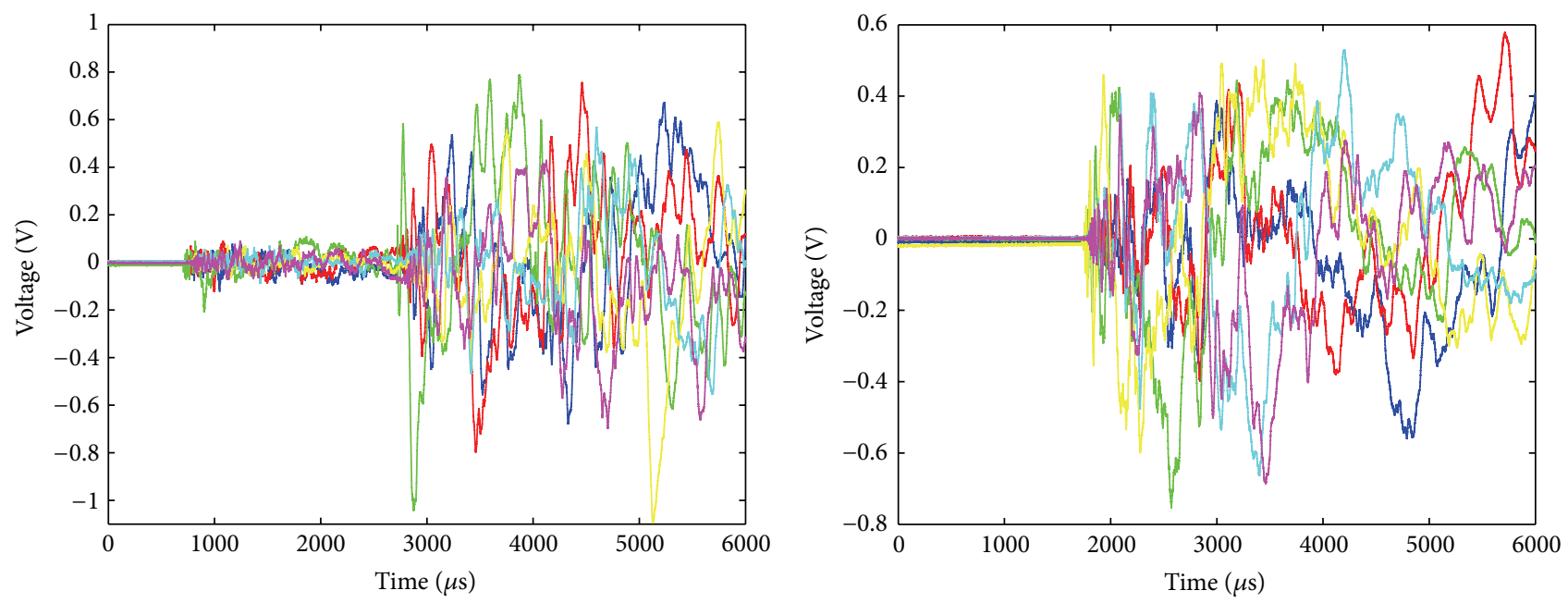

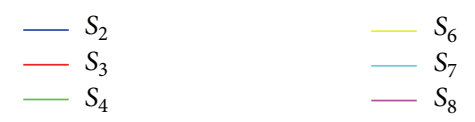

(a)

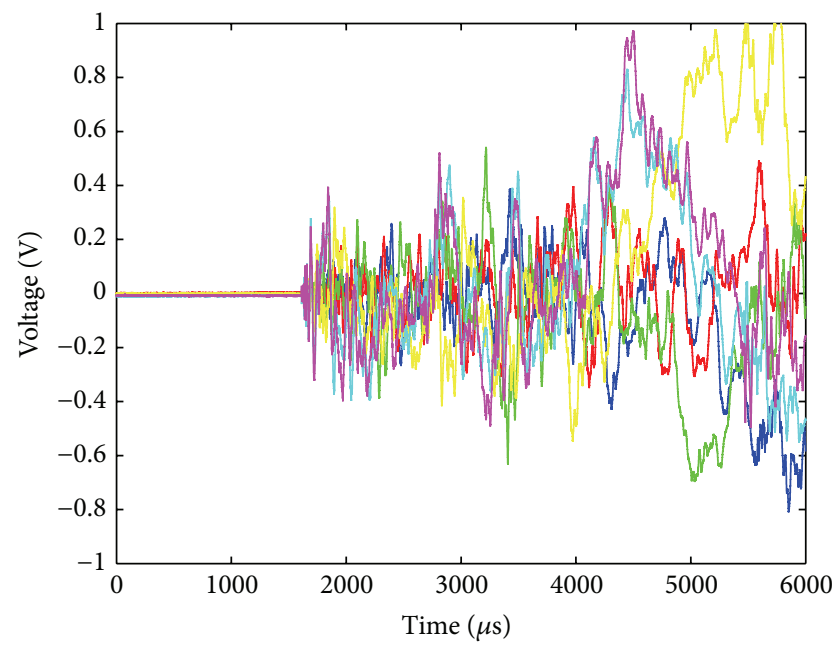

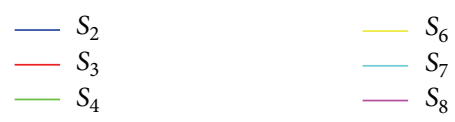

(b)

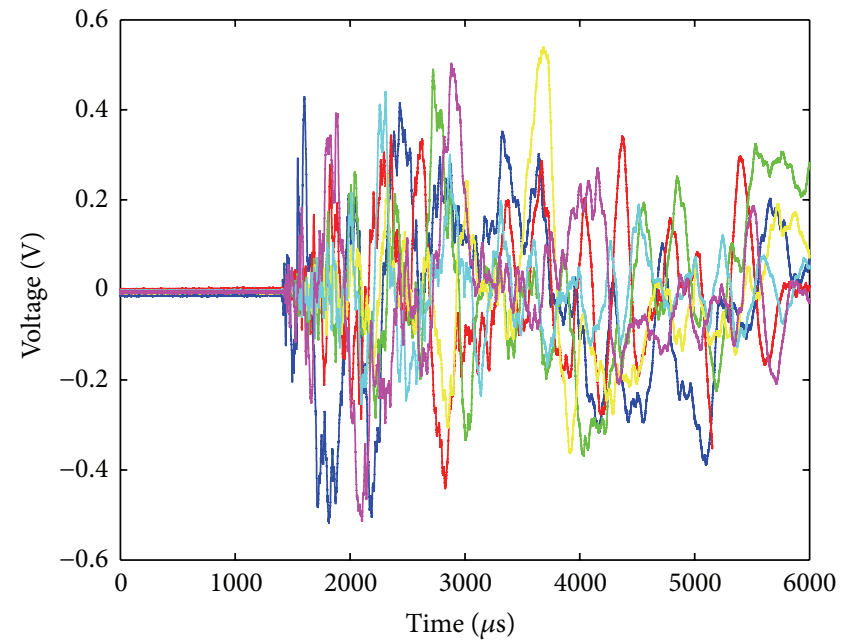

(c)

$S_{6}$
$-S_{7}$
$-S_{8}$

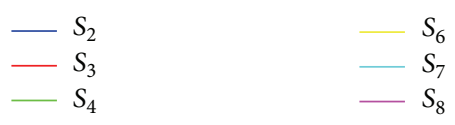

(d)

FIgUre 7: AE wave signals for simulated AE events (a) $\mathrm{PLB}_{2}$, (b) $\mathrm{PLB}_{3}$, (c) $\mathrm{PLB}_{4}$, and (d) $\mathrm{PLB}_{5}$.

TABLE 3: CWT measured time difference data at $20 \mathrm{kHz}$.

\begin{tabular}{lccccc}
\hline & $\Delta t_{24}(\mu \mathrm{s})$ & $\Delta t_{34}(\mu \mathrm{s})$ & $\Delta t_{64}(\mu \mathrm{s})$ & $\Delta t_{74}(\mu \mathrm{s})$ & 84.1 \\
\hline $\mathrm{PLB}_{1}$ & 43.7 & 82.0 & 47.8 & 179.5 & 245.8 \\
$\mathrm{PLB}_{2}$ & 100.9 & 84.4 & -21.1 & 74.4 & -1.0 \\
$\mathrm{PLB}_{3}$ & 147.4 & 152.2 & -115.9 & -144.9 & 71.9 \\
$\mathrm{PLB}_{4}$ & 40.0 & 106.3 & 65.8 & 67.5 \\
$\mathrm{PLB}_{5}$ & -154.0 & -36.0 & & -138.4 \\
\hline
\end{tabular}



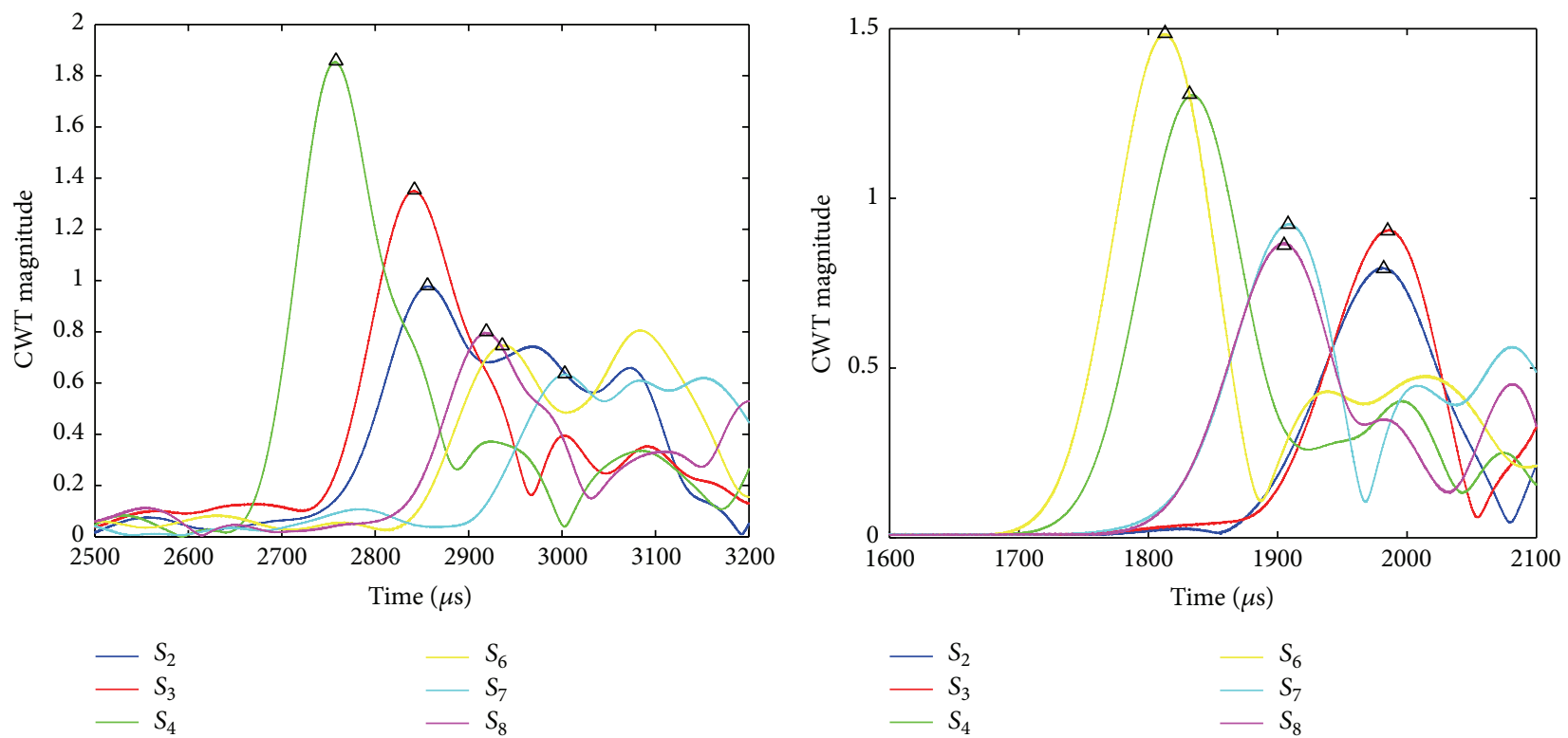

(a)
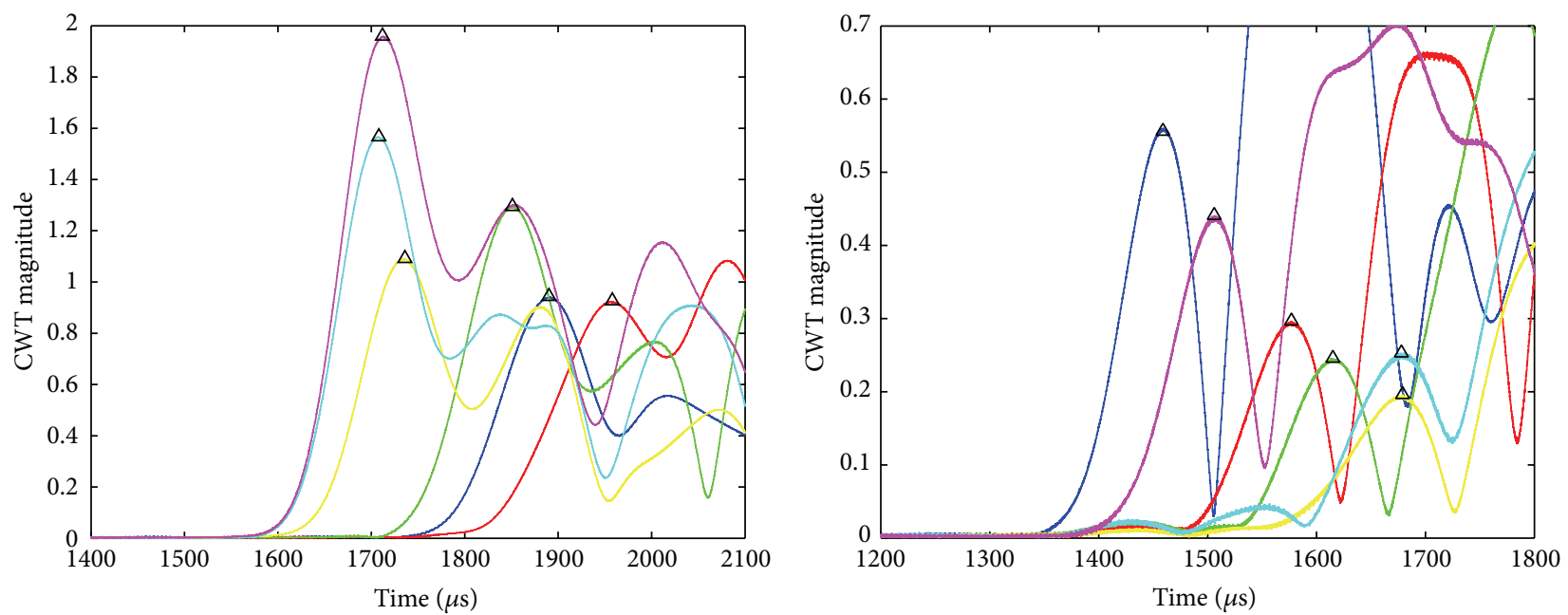

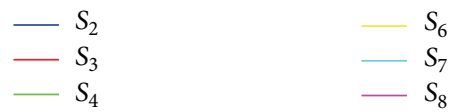

(c)
$S_{6}$
$-S_{7}$

$-S_{7}$

)

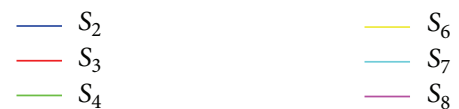

(d)

FIgure 8: CWT of AE wave signals at $20 \mathrm{kHz}$ for simulated AE events (a) $\mathrm{PLB}_{2}$, (b) $\mathrm{PLB}_{3}$, (c) PLB , and (d) $\mathrm{PLB}_{5}$.

For the initial values, the $x$-coordinate of the AE source location is uniformly sampled from $[-150,150] \mathrm{mm}$, the $y$ coordinate of the AE source location is uniformly sampled from $[-200,200] \mathrm{mm}$, and the wave velocity at $20 \mathrm{kHz}$ are uniformly sampled from $[0,3000] \mathrm{m} / \mathrm{s}$. Figures 5(a) and 5(b) show the samples of $x-y$ coordinates of the AE source location by MCMC, and Figure 5(c) shows the simultaneously sampled wave velocity of the AE wave at $20 \mathrm{kHz}$ for the simulated $\mathrm{AE}$ event $\mathrm{PLB}_{1}$. For each parameter, 100000 samples in total are obtained and the first 40000 samples are set as the "burn-in" period. Gaussian distributions are used to fit these samples. Figure 6 illustrates the fitted joint distribution of $x-y$ coordinates of the AE source location in 2D contour view for the simulated $A E$ event $\mathrm{PLB}_{1}$. The actual $\mathrm{PLB}_{1}$ source location is also labeled in Figure 6 for comparison. The means of the fitted distributions of the unknown parameters are output as their estimates. For $\mathrm{PLB}_{1}$, the identified AE source location is $(0.4,-2.0) \mathrm{mm}$; the distance between the identified AE source location and the actual one is less than $3.0 \mathrm{~mm}$ with a relative error of $1.4 \%$ with respect to the minimum sensor spacing $(150 \mathrm{~mm})$, demonstrating the effectiveness of the proposed Bayesian AE source localization approach. For the wave velocity, the identified value at $20 \mathrm{kHz}$ is $1187.8 \mathrm{~m} / \mathrm{s}$, which is close to the theoretical value $1200 \mathrm{~m} / \mathrm{s}$ calculated 


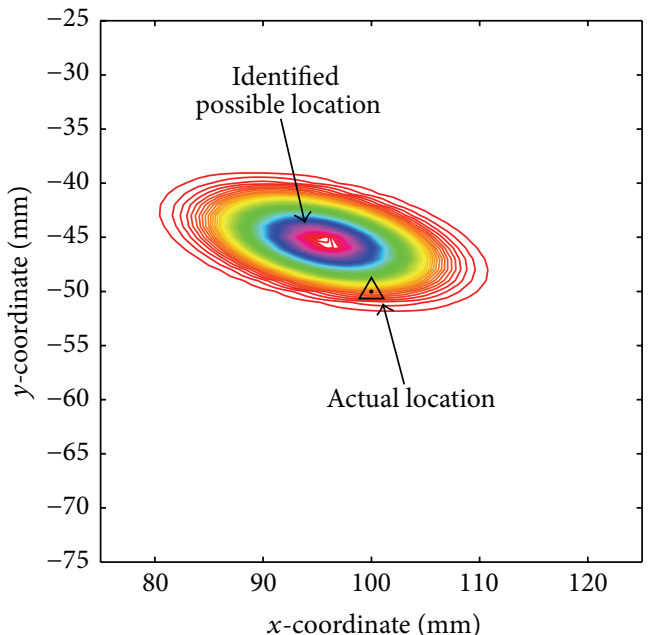

(a)

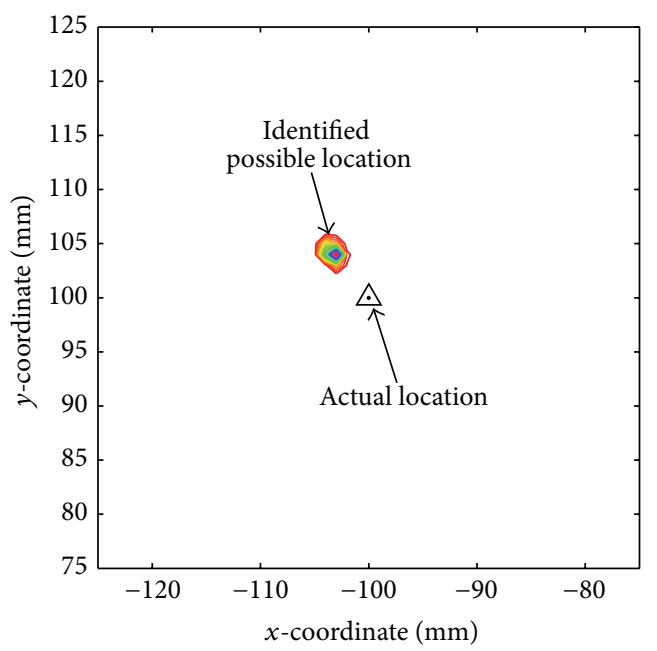

(c)
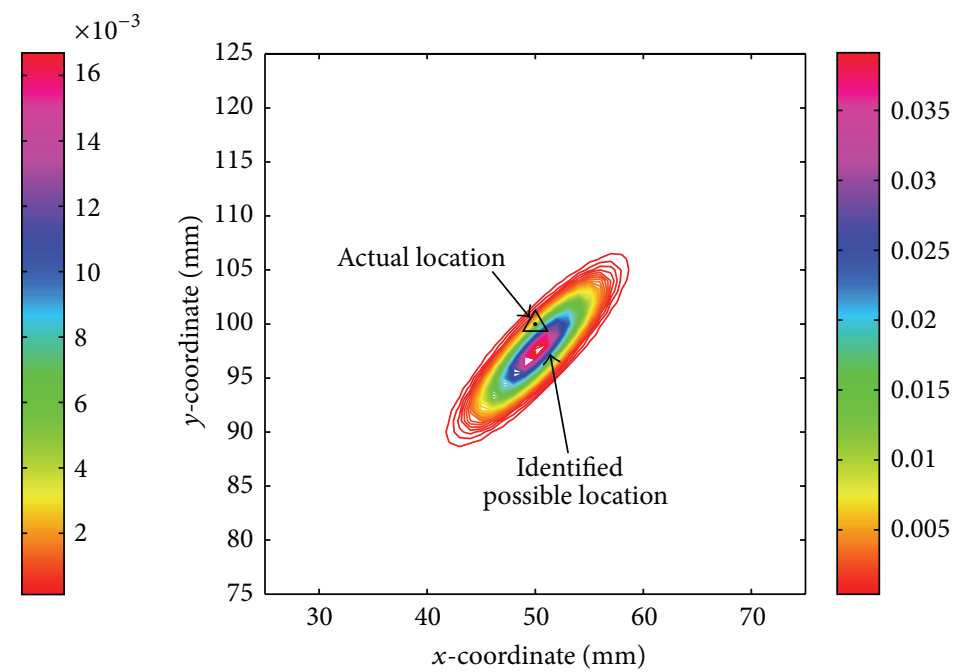

(b)
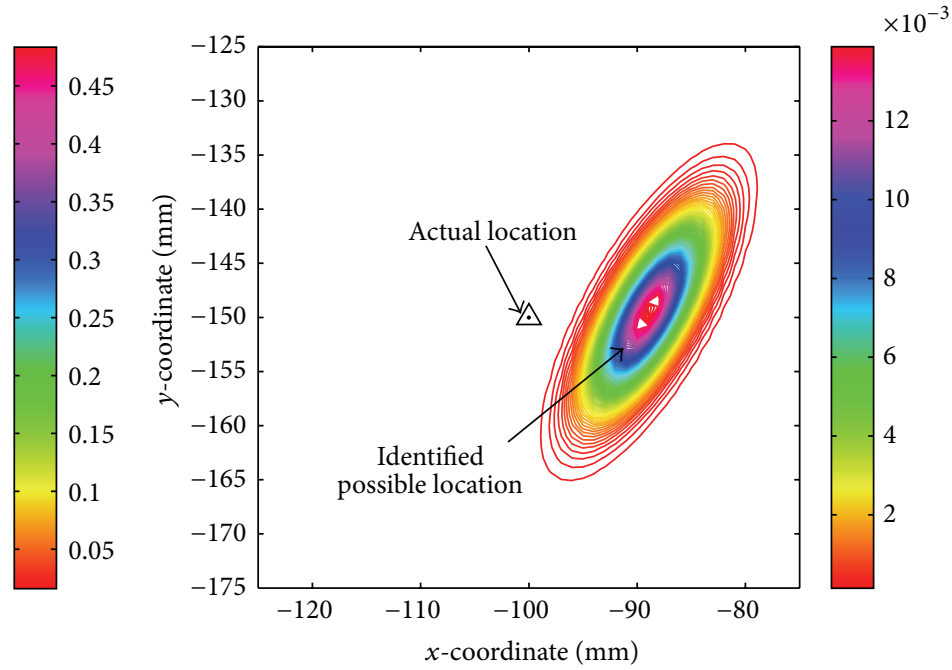

(d)

Figure 9: Identified source location for simulated AE events by time difference data at $20 \mathrm{kHz}$ (a) $\mathrm{PLB}_{2}$, (b) $\mathrm{PLB}_{3}$, (c) $\mathrm{PLB}_{4}$, and (d) $\mathrm{PLB}_{5}$.

by freeware "Vallen Dispersion" contained in "AGU-Vallen Wavelet" package provided by the AE company Vallen System [40], with nominal material properties for the stiffened aluminum panel as Young's modulus $73 \mathrm{GPa}$, Poisson's ratio 0.32 , and density $2790 \mathrm{~kg} / \mathrm{m}^{3}$. It should be noted that, due to the various uncertainties involved, these nominal material properties are not exactly the same as those for this specific stiffened aluminum panel; thus the theoretical wave velocity value is only used for reference. The results also give the standard deviations and correlation coefficient for the $\mathrm{AE}$ source location and the wave velocity as listed in Table 1 , which can be used for uncertainty analysis.

Similar identification results are obtained for the other four PLB events. Figures 7(a)-7(d) show the AE wave signals emitted by the simulated $\mathrm{AE}$ events $\mathrm{PLB}_{2}-\mathrm{PLB}_{5}$. Figures $8(\mathrm{a})-8(\mathrm{~d})$ show the magnitudes of CWT coefficients of the $\mathrm{AE}$ wave signals at frequency of $20 \mathrm{kHz}$. The corresponding time arrivals of the $\mathrm{AE}$ wave signals at $20 \mathrm{kHz}$ are determined and labeled by triangles in Figure 8. The CWT measured time difference data at $20 \mathrm{kHz}$ for these four cases are listed in Table 3. The same MCMC procedure as presented for $\mathrm{PLB}_{1}$ case is then performed for these four PLB cases. The identification results are listed in Table 4 together with those for $\mathrm{PLB}_{1}$. Figures 9(a)-9(d) illustrate the fitted joint distributions of $x-y$ coordinates of the identified AE source locations for these four cases, respectively. The actual PLB locations are also labeled in Figure 9 for comparison. Figure 10(a) shows the identified wave velocities compared with the theoretical one at $20 \mathrm{kHz}$ for the five PLB events; error bars with three times of standard deviations are plotted with the mean values. From Table 4 and Figures 6 and 9 , it can be seen that the identified mean values of the simulated AE source locations are quite close to the actual ones. In addition to the point estimates, the Bayesian approach also provides interval estimates for uncertainty analysis. 


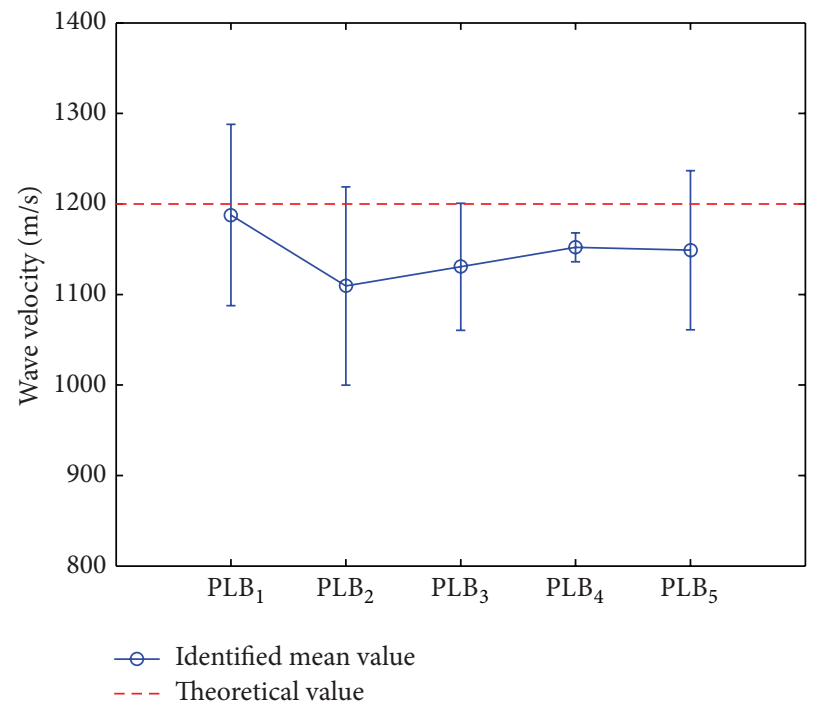

(a)

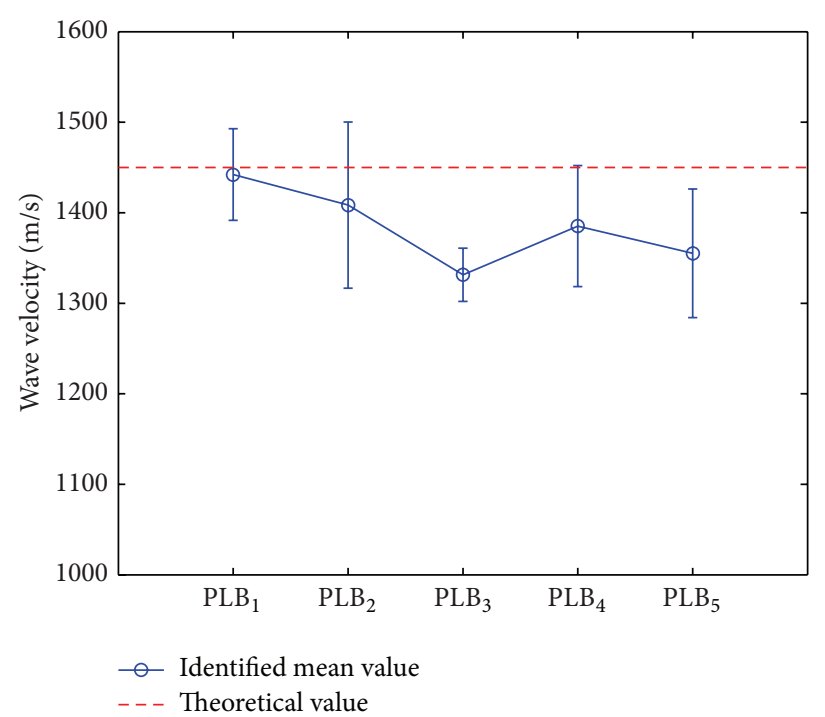

(b)

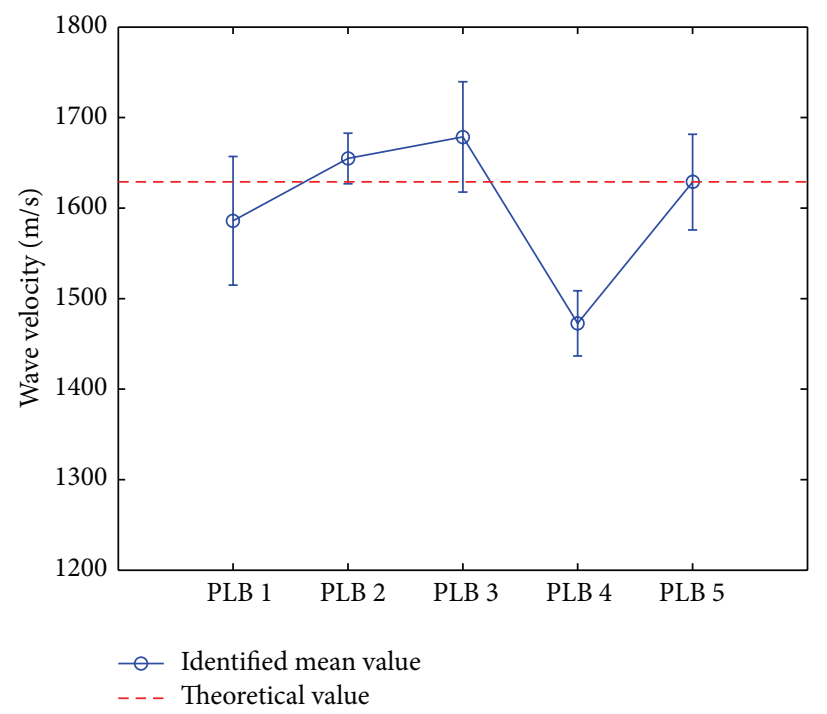

(c)

FIgURE 10: Identified wave velocities for the simulated AE events $\mathrm{PLB}_{1}-\mathrm{PLB}_{5}$ (a) $20 \mathrm{kHz}$, (b) $30 \mathrm{kHz}$, and (c) $40 \mathrm{kHz}$.

TABLE 4: Identified results by CWT measured time difference data at $20 \mathrm{kHz}$.

\begin{tabular}{lccccc}
\hline & & \multicolumn{2}{c}{ Source location } & \multicolumn{2}{c}{ Wave velocity } \\
& $\left(\bar{x}_{d}, \bar{y}_{d}\right)(\mathrm{mm})$ & $\left(\sigma_{x}, \sigma_{y}\right)(\mathrm{mm})$ & $\rho$ & $\bar{V}_{g}(\mathrm{~m} / \mathrm{s})$ & $\sigma_{V}(\mathrm{~m} / \mathrm{s})$ \\
\hline $\mathrm{PLB}_{1}$ & $(0.4,-2.0)$ & $(1.5,1.9)$ & 0.2 & 1.4 & 33.4 \\
$\mathrm{PLB}_{2}$ & $(95.6,-45.4)$ & $(5.0,2.1)$ & -0.4 & 4.3 & 1187.8 \\
$\mathrm{PLB}_{3}$ & $(50.2,97.6)$ & $(2.8,2.9)$ & 0.9 & 1.6 & 1109.4 \\
$\mathrm{PLB}_{4}$ & $(-103.3,104.1)$ & $(0.5,0.5)$ & -0.4 & 3.5 & 1130.6 \\
$\mathrm{PLB}_{5}$ & $(-88.2,-149.5)$ & $(3.3,5.1)$ & 0.7 & 7.9 & 1148.9 \\
\hline
\end{tabular}

As aforementioned, one appealing property of CWT for time-frequency analysis of $\mathrm{AE}$ wave signals is that it can obtain TOF information of the AE waves at different frequencies without any difficulties. To further demonstrate the effectiveness of the proposed $\mathrm{AE}$ source localization approach, we take time difference data at other two frequencies, that is, $30 \mathrm{kHz}$ and $40 \mathrm{kHz}$, to localize the AE sources. The CWT measured time differences of AE waves at $30 \mathrm{kHz}$ and $40 \mathrm{kHz}$ in these five PLB cases are listed in Tables 5 and 6 , respectively. Tables 7 and 8 present the identified AE 


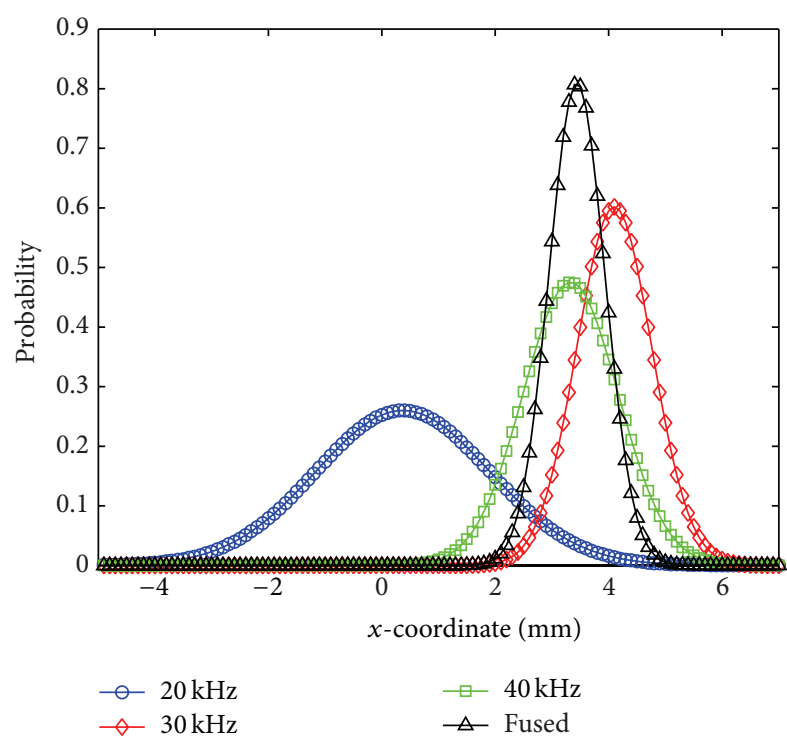

(a)

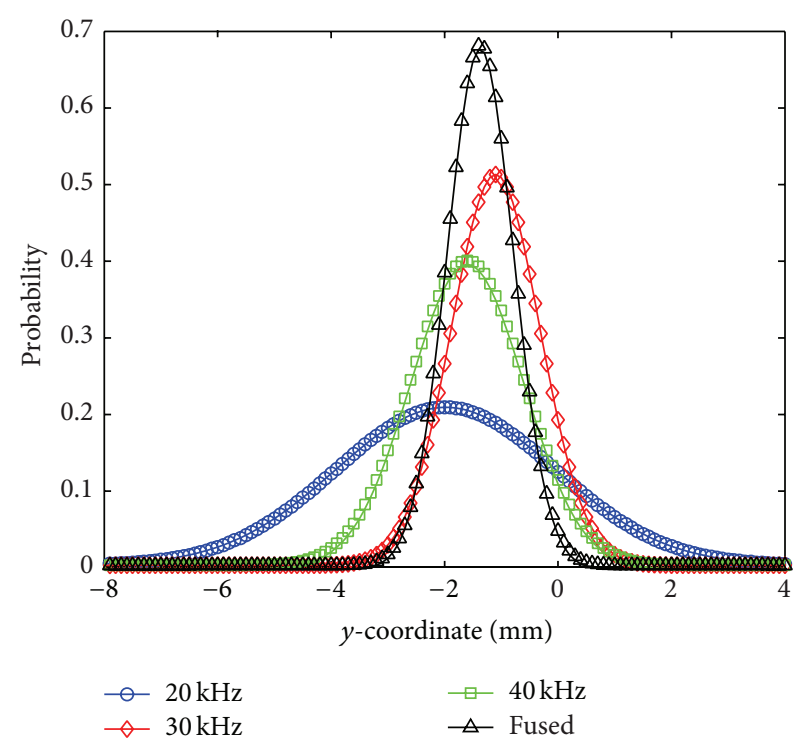

(b)

FIGURE 11: Merged distribution for $x$ - and $y$-coordinates of source location for simulated AE event PLB $_{1}$.

TABLE 5: CWT measured time difference data at $30 \mathrm{kHz}$.

\begin{tabular}{lccccc}
\hline & $\Delta t_{24}(\mu \mathrm{s})$ & $\Delta t_{34}(\mu \mathrm{s})$ & $\Delta t_{64}(\mu \mathrm{s})$ & $\Delta t_{74}(\mu \mathrm{s})$ & 73.3 \\
\hline $\mathrm{PLB}_{1}$ & 36.7 & 70.4 & 39.7 & 201.4 & 5.8 \\
$\mathrm{PLB}_{2}$ & 76.5 & 63.0 & 144.5 & 58.2 & 135.3 \\
$\mathrm{PLB}_{3}$ & 115.9 & 130.0 & -21.9 & -110.0 & 56.4 \\
$\mathrm{PLB}_{4}$ & 35.7 & 87.9 & -89.6 & 52.7 & -111.1 \\
$\mathrm{PLB}_{5}$ & -124.3 & -25.9 & 56.4 & -91.6 \\
\hline
\end{tabular}

TABLE 6: CWT measured time difference data at $40 \mathrm{kHz}$.

\begin{tabular}{lccccc}
\hline & $\Delta t_{24}(\mu \mathrm{s})$ & $\Delta t_{34}(\mu \mathrm{s})$ & $\Delta t_{64}(\mu \mathrm{s})$ & $\Delta t_{74}(\mu \mathrm{s})$ & 66.9 \\
\hline $\mathrm{PLB}_{1}$ & 31.1 & 64.4 & 34.4 & 178.0 & $5 t_{84}(\mu \mathrm{s})$ \\
$\mathrm{PLB}_{2}$ & 71.7 & 54.5 & 125.9 & 52.7 & 120.5 \\
$\mathrm{PLB}_{3}$ & 99.7 & 105.2 & -18.5 & -104.4 & 52.4 \\
$\mathrm{PLB}_{4}$ & 32.0 & 80.9 & -83.5 & 36.7 & -108.5 \\
$\mathrm{PLB}_{5}$ & -112.4 & -23.7 & 46.5 & -82.4 \\
\hline
\end{tabular}

source locations and corresponding wave velocities at $30 \mathrm{kHz}$ and $40 \mathrm{kHz}$, respectively. Figures $10(\mathrm{~b})$ and $10(\mathrm{c})$ show the identified wave velocities at $30 \mathrm{kHz}$ and $40 \mathrm{kHz}$ compared with the theoretical ones, respectively.

\subsubsection{Fused Results for Multiple-Frequency Data. After AE} source localization results from time difference data at different frequencies, that is, $20 \mathrm{kHz}, 30 \mathrm{kHz}$, and $40 \mathrm{kHz}$ in the present study, are obtained and fitted by two-dimensional Gaussian distributions; the proposed data fusion scheme described in Section 2.4 can be applied to get the final fused results. Figure 11 shows the merging of three individual distributions obtained by time difference data at three frequencies into one distribution for $x$ - and $y$-coordinates of the source location for simulated AE event $\mathrm{PLB}_{1}$. Figure 12(a) illustrates the fused joint distribution of $x-y$ coordinates of the $\mathrm{AE}$ source location in $2 \mathrm{D}$ contour view corresponding to Figure 11. The actual $\mathrm{PLB}_{1}$ source location is also labeled in Figure 12(a) for comparison. Figures 12(b)-12(e) illustrate the fused joint distributions of $x-y$ coordinates of the identified AE source locations for the rest of the four cases, respectively. Also these fuse AE source localization results are listed in Table 9. From Figure 12 and Table 9, it can be seen that, compared with the individually obtained localization results, for most of the cases, the fused results have mean values closer to the actual ones and narrower standard deviations, demonstrating that the data fusion scheme indeed can increase the accuracy of the AE source localization results and reduce their associated uncertainties. 


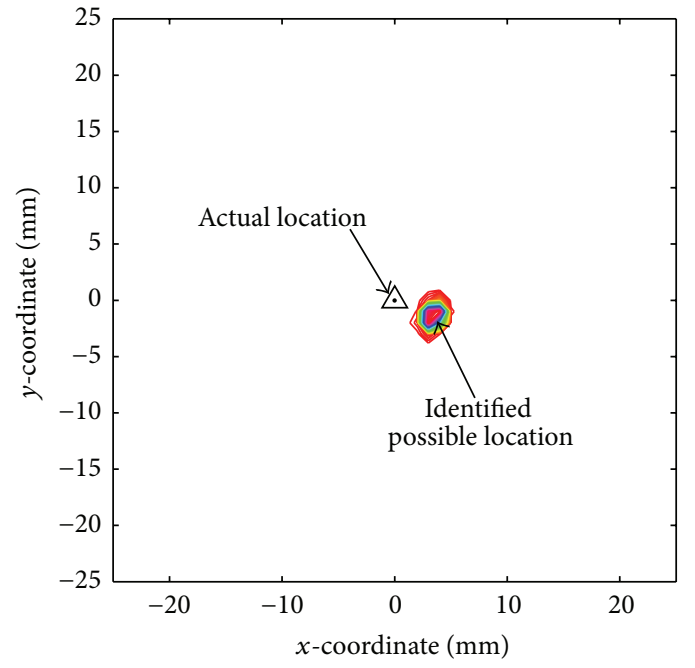

(a)

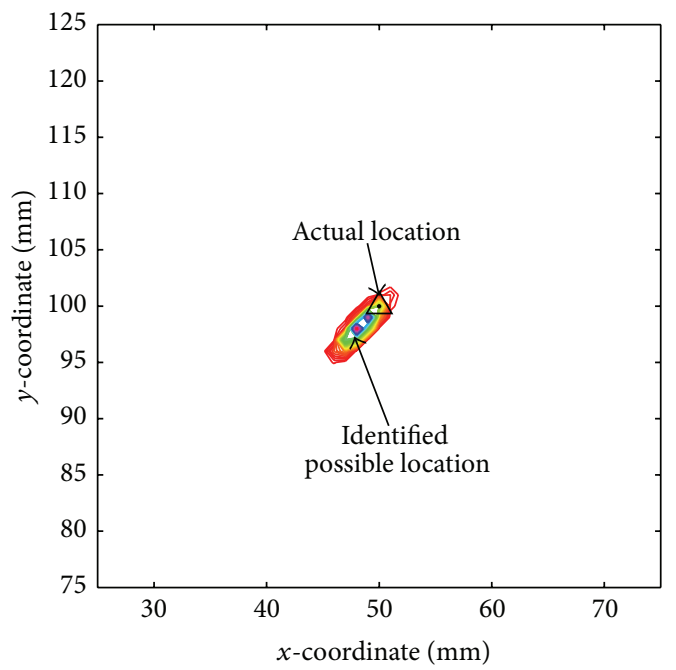

(c)
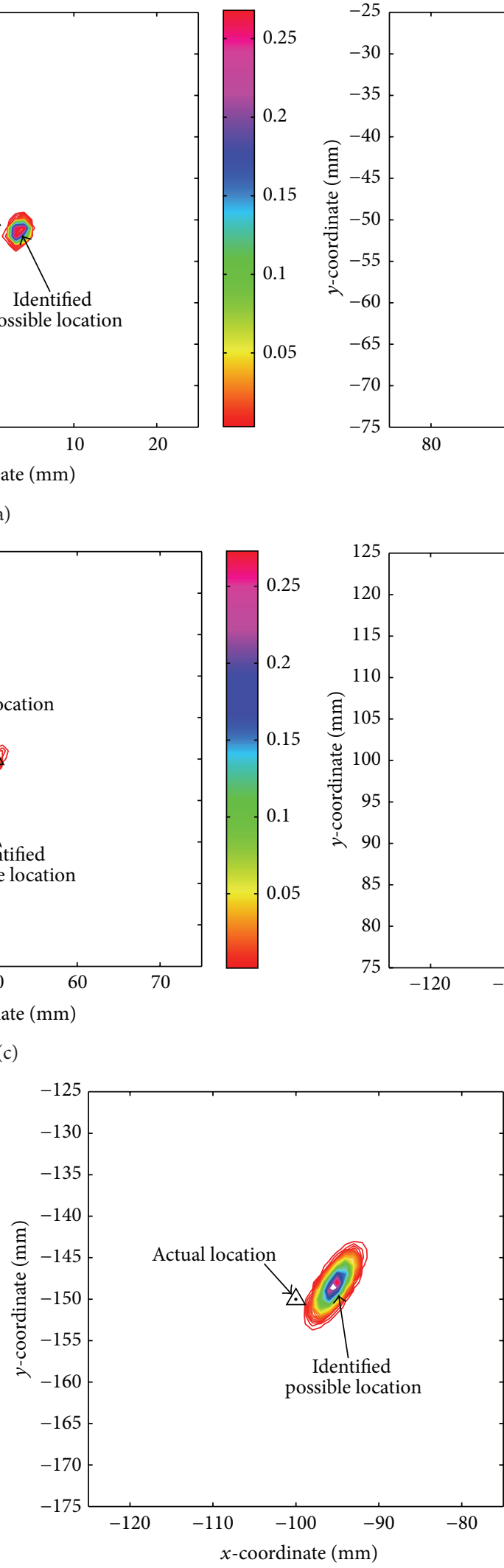

(e)

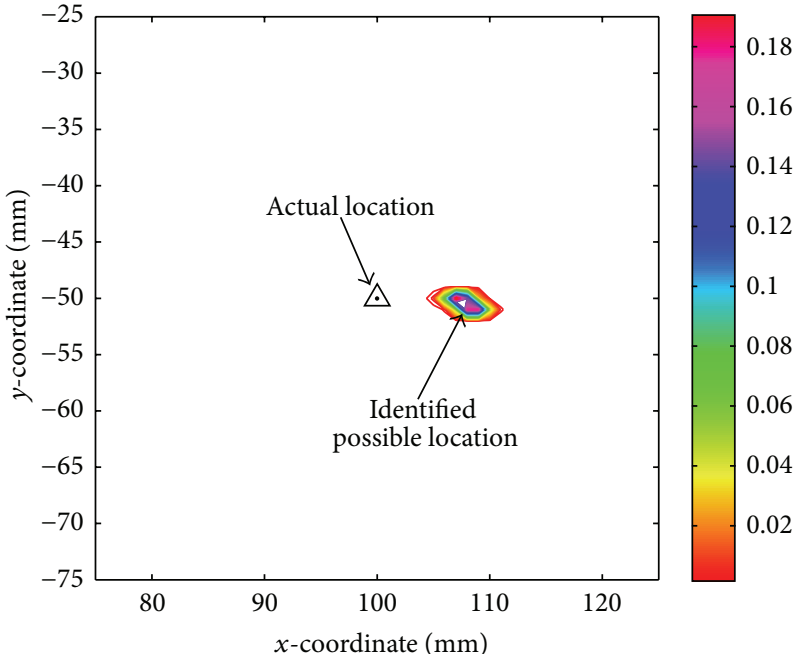

(b)

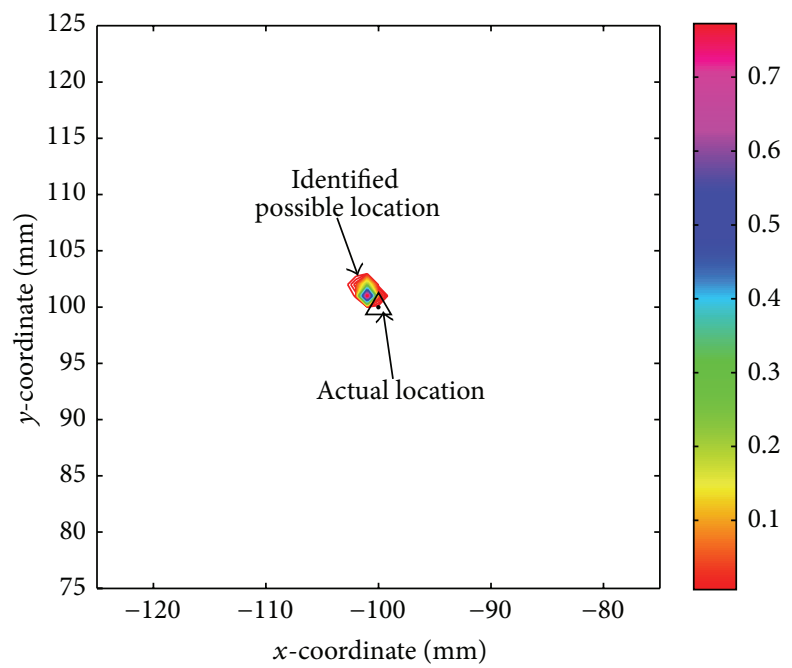

(d)

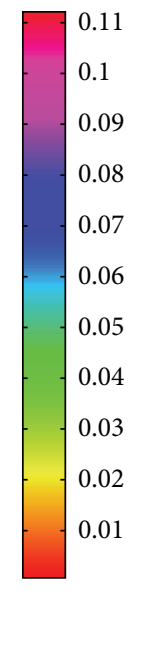

Figure 12: Identified source location for simulated AE events after data fusion (a) $\mathrm{PLB}_{1}$, (b) $\mathrm{PLB}_{2}$, (c) $\mathrm{PLB}_{3}$, (d) $\mathrm{PLB}_{4}$, and (e) $\mathrm{PLB}_{5}$. 
TABLE 7: Identified results by CWT measured time difference data at $30 \mathrm{kHz}$.

\begin{tabular}{lccccc}
\hline & & \multicolumn{2}{c}{ Source location } & & \multicolumn{2}{c}{ Wave velocity } \\
& $\left(\bar{x}_{d}, \bar{y}_{d}\right)(\mathrm{mm})$ & $\left(\sigma_{x}, \sigma_{y}\right)(\mathrm{mm})$ & $\rho$ & Error $(\%)$ & $\bar{V}_{g}(\mathrm{~m} / \mathrm{s})$ \\
\hline $\mathrm{PLB}_{1}$ & $(4.1,-1.1)$ & $(0.7,0.8)$ & 0.2 & 2.8 & 1442.2 \\
$\mathrm{PLB}_{2}$ & $(102.5,-51.1)$ & $(3.5,1.5)$ & -0.4 & 1.8 & 1408.5 \\
$\mathrm{PLB}_{3}$ & $(44.3,95.7)$ & $(1.3,1.5)$ & 0.9 & 4.8 & 13.9 \\
$\mathrm{PLB}_{4}$ & $(-96.9,98.7)$ & $(0.8,0.8)$ & -0.3 & 2.2 & 1331.5 \\
$\mathrm{PLB}_{5}$ & $(-88.0,-141.4)$ & $(2.0,3.0)$ & 0.7 & 9.8 & 1385.2 \\
\hline
\end{tabular}

TABLE 8: Identified results by CWT measured time difference data at $40 \mathrm{kHz}$.

\begin{tabular}{lccccc}
\hline & & \multicolumn{2}{c}{ Source location } & & \multicolumn{2}{c}{ Wave velocity } \\
& $\left(\bar{x}_{d}, \bar{y}_{d}\right)(\mathrm{mm})$ & $\left(\sigma_{x}, \sigma_{y}\right)(\mathrm{mm})$ & $\rho$ & Error $(\%)$ & $\bar{V}_{g}(\mathrm{~m} / \mathrm{s})$ \\
\hline $\mathrm{PLB}_{1}$ & $(3.3,-1.6)$ & $(0.8,1.0)$ & 0.2 & 2.4 & 1586.0 \\
$\mathrm{PLB}_{2}$ & $(108.9,-50.7)$ & $(1.0,0.4)$ & -0.5 & 6.0 & 1654.8 \\
$\mathrm{PLB}_{3}$ & $(54.7,103.2)$ & $(1.8,1.8)$ & 0.9 & 3.8 & 1678.6 \\
$\mathrm{PLB}_{4}$ & $(-100.1,96.6)$ & $(0.9,0.9)$ & -0.4 & 2.3 & 1472.7 \\
$\mathrm{PLB}_{5}$ & $(-102.3,-153.9)$ & $(1.7,2.4)$ & 0.8 & 3.0 & 1628.7 \\
\hline
\end{tabular}

TABLE 9: AE source location results after data fusion.

\begin{tabular}{lcccc}
\hline \multicolumn{5}{c}{ Source location } \\
& $\left(\bar{x}_{d}, \bar{y}_{d}\right)(\mathrm{mm})$ & $\left(\sigma_{x}, \sigma_{y}\right)(\mathrm{mm})$ & $\rho$ & Error $(\%)$ \\
\hline $\mathrm{PLB}_{1}$ & $(3.4,-1.3)$ & $(0.5,0.6)$ & 0.2 & 2.4 \\
$\mathrm{PLB}_{2}$ & $(107.9,-50.5)$ & $(0.9,0.4)$ & -0.4 & 5.3 \\
$\mathrm{PLB}_{3}$ & $(48.4,98.2)$ & $(1.0,1.1)$ & 0.8 & 1.6 \\
$\mathrm{PLB}_{4}$ & $(-101.0,101.3)$ & $(0.4,0.4)$ & -0.4 & 1.1 \\
$\mathrm{PLB}_{5}$ & $(-95.3,-148.3)$ & $(1.2,1.7)$ & 0.8 & 3.3 \\
\hline
\end{tabular}

\section{Conclusions}

In this paper, a Bayesian approach for localizing AE source in plate-like structures is proposed, while considering uncertainties from modeling error and measurement noise. After the TOF information of the AE wave signals in each sensor channel is measured by CWT, a Bayesian procedure for identification of the unknown $\mathrm{AE}$ source location, wave velocity, and their associated uncertainties is developed and MCMC algorithm is employed to realize the identification process to draw samples from their posterior distributions. A data fusion scheme is also introduced to fuse results by merging individually identified distributions obtained by time difference data at multiple frequencies.

Experimental studies on a stiffened aluminum panel with AE events simulated by PLBs have demonstrated that, rather than pinpointing the $\mathrm{AE}$ source location in a single point by traditional deterministic triangulation approach, the proposed Bayesian approach can provide a probabilistic description of the $\mathrm{AE}$ source location and quantify the uncertainties of the identification results. With data fusion, the means of the posterior distributions of the AE source locations are quite close to the actual ones, and the uncertainties of the results are reduced, demonstrating the effectiveness of the proposed Bayesian AE source localization approach.

\section{Conflict of Interests}

The authors declare that there is no conflict of interests regarding the publication of this paper.

\section{Acknowledgments}

This research is supported by the Research Fund for the Doctoral Program of Higher Education of China under Grant no. 20113218120004, by the Natural Science Foundation of Jiangsu Province under Grant no. BK20130783, and by the Fundamental Research Funds for the Central Universities under Grant no. NS2012138.

\section{References}

[1] M. J. Eaton, R. Pullin, and K. M. Holford, "Towards improved damage location using acoustic emission," Proceedings of the Institution of Mechanical Engineers, Part C: Journal of Mechanical Engineering Science, vol. 226, no. 9, pp. 2141-2153, 2012.

[2] W. J. Staszewski, S. Mahzan, and R. Traynor, "Health monitoring of aerospace composite structures-active and passive approach," Composites Science and Technology, vol. 69, no. 11-12, pp. 1678-1685, 2009.

[3] J. Haywood, P. T. Coverley, W. J. Staszewski, and K. Worden, "An automatic impact monitor for a composite panel employing smart sensor technology," Smart Materials and Structures, vol. 14, no. 1, pp. 265-271, 2005.

[4] J. R. LeClerc, K. Worden, W. J. Staszewski, and J. Haywood, "Impact detection in an aircraft composite panel-a neuralnetwork approach," Journal of Sound and Vibration, vol. 299, no. 3, pp. 672-682, 2007.

[5] H. Fu and Q. Xu, "Locating impact on structural plate using principal component analysis and support vector machines," Mathematical Problems in Engineering, vol. 2013, Article ID 352149, 8 pages, 2013.

[6] B. Park, H. Sohn, S. E. Olson, M. P. DeSimio, K. S. Brown, and M. M. Derriso, "Impact localization in complex structures using 
laser-based time reversal," Structural Health Monitoring, vol. 11, no. 5, pp. 577-588, 2012.

[7] T. He, Q. Pan, Y. Liu, X. Liu, and D. Hu, "Near-field beamforming analysis for acoustic emission source localization," Ultrasonics, vol. 52, no. 5, pp. 587-592, 2012.

[8] R. Gangadharan, G. Prasanna, M. R. Bhat, C. R. L. Murthy, and S. Gopalakrishnan, "Acoustic emission source location in composite structure by Voronoi construction using geodesic curve evolution," The Journal of the Acoustical Society of America, vol. 126, no. 5, pp. 2324-2330, 2009.

[9] A. Tobias, "Acoustic-emission source location in two dimensions by an array of three sensors," Non-Destructive Testing, vol. 9, no. 1, pp. 9-12, 1976.

[10] L. Gaul and S. Hurlebaus, "Identification of the impact location on a plate using wavelets," Mechanical Systems and Signal Processing, vol. 12, no. 6, pp. 783-795, 1998.

[11] F. Ciampa and M. Meo, "Acoustic emission source localization and velocity determination of the fundamental mode $A_{0}$ using wavelet analysis and a Newton-based optimization technique," Smart Materials and Structures, vol. 19, no. 4, Article ID 045027, 2010.

[12] T. Kundu, H. Nakatani, and N. Takeda, "Acoustic source localization in anisotropic plates," Ultrasonics, vol. 52, no. 6, pp. 740746, 2012.

[13] P. T. Coverley and W. J. Staszewski, "Impact damage location in composite structures using optimized sensor triangulation procedure," Smart Materials and Structures, vol. 12, no. 5, pp. 795-803, 2003.

[14] M. Meo, G. Zumpano, M. Piggott, and G. Marengo, "Impact identification on a sandwich plate from wave propagation responses," Composite Structures, vol. 71, no. 3-4, pp. 302-306, 2005.

[15] B. Castagnede, W. Sachse, and K. Y. Kim, "Location of pointlike AE sources in anisotropic plates," The Journal of the Acoustical Society of America, vol. 86, no. 3, pp. 1161-1171, 1989.

[16] S. M. Ziola and M. R. Gorman, "Source location in thin plates using cross-correlation," The Journal of the Acoustical Society of America, vol. 90, no. 5, pp. 2551-2556, 1991.

[17] P. Sedlak, Y. Hirose, and M. Enoki, "Acoustic emission localization in thin multi-layer plates using first-arrival determination," Mechanical Systems and Signal Processing, vol. 36, no. 2, pp. 636649, 2013.

[18] H. Jeong and Y.-S. Jang, "Wavelet analysis of plate wave propagation in composite laminates," Composite Structures, vol. 49, no. 4, pp. 443-450, 2000.

[19] M. A. Hamstad, A. O'Gallagher, and J. Gary, "A wavelet transform applied to acoustic emission signals: part 1: source identification," Journal of Acoustic Emission, vol. 20, no. 1, pp. 39-61, 2002.

[20] E. D. Niri and S. Salamone, "A probabilistic framework for acoustic emission source localization in plate-like structures," Smart Materials and Structures, vol. 21, no. 3, Article ID 035009, 2012.

[21] E. D. Dehghan Niri, A. Farhidzadeh, and S. Salamone, "Adaptive multisensor data fusion for acoustic emission source localization in noisy environment," Structural Health Monitoring, vol. 12, no. 1, pp. 59-77, 2013.

[22] M. Kaphle, A. C. C. Tan, D. P. Thambiratnam, and T. H. T. Chan, "Identification of acoustic emission wave modes for accurate source location in plate-like structures," Structural Control and Health Monitoring, vol. 19, no. 2, pp. 187-198, 2012.
[23] A. Perelli, L. de Marchi, A. Marzani, and N. Speciale, "Acoustic emission localization in plates with dispersion and reverberations using sparse PZT sensors in passive mode," Smart Materials and Structures, vol. 21, no. 2, Article ID 025010, 2012.

[24] J. C. Dodson and D. J. Inman, “Thermal sensitivity of Lamb waves for structural health monitoring applications," Ultrasonics, vol. 53, no. 3, pp. 677-685, 2013.

[25] T. Schumacher, D. Straub, and C. Higgins, "Toward a probabilistic acoustic emission source location algorithm: a Bayesian approach," Journal of Sound and Vibration, vol. 331, no. 19, pp. 4233-4245, 2012.

[26] G. Yan, "A Bayesian approach for damage localization in platelike structures using Lamb waves," Smart Materials and Structures, vol. 22, no. 3, Article ID 035012, 17 pages, 2013.

[27] I. A. Viktorov, Rayleigh and Lamb Waves, Plenum Press, New York, NY, USA, 1967.

[28] R. D. Mindlin, "Influence of rotary inertia and shear on flexural motions of isotropic elastic plates," Journal of Applied Mechanics, vol. 18, pp. 31-38, 1951.

[29] J. L. Beck and L. S. Katafygiotis, "Updating models and their uncertainties. I: Bayesian statistical framework," ASCE Journal of Engineering Mechanics, vol. 124, no. 4, pp. 455-461, 1998.

[30] J. L. Beck and S.-K. Au, "Bayesian updating of structural models and reliability using Markov chain Monte Carlo simulation," ASCE Journal of Engineering Mechanics, vol. 128, no. 4, pp. 380391, 2002.

[31] C. T. Ng, M. Veidt, and H. F. Lam, "Guided wave damage characterisation in beams utilising probabilistic optimisation," Engineering Structures, vol. 31, no. 12, pp. 2842-2850, 2009.

[32] T. Yin, H.-F. Lam, and H.-M. Chow, "A Bayesian probabilistic approach for crack characterization in plate structures," Computer-Aided Civil and Infrastructure Engineering, vol. 25, no. 5, pp. 375-386, 2010.

[33] J. M. Nichols, W. A. Link, K. D. Murphy, and C. C. Olson, "A Bayesian approach to identifying structural nonlinearity using free-decay response: application to damage detection in composites," Journal of Sound and Vibration, vol. 329, no. 15, pp. 2995-3007, 2010.

[34] R. Sternfels and C. J. Earls, "Reduced-order model tracking and interpolation to solve PDE-based Bayesian inverse problems," Inverse Problems, vol. 29, no. 7, Article ID 075014, 19 pages, 2013.

[35] W. L. Dunn and J. K. Shultis, Exploring Monte Carlo Methods, Elsevier, Amsterdam, The Netherlands, 2011.

[36] Z. Su, X. Wang, L. Cheng, L. Yu, and Z. Chen, "On selection of data fusion schemes for structural damage evaluation," Structural Health Monitoring, vol. 8, no. 3, pp. 223-241, 2009.

[37] R. C. Smith and P. Cheeseman, "On the representation and estimation of spatial uncertainty," The International Journal of Robotics Research, vol. 5, no. 4, pp. 56-68, 1986.

[38] A. W. Stroupe, M. Martin, and T. Balch, "Merging Gaussian distributions for object localization in multi-robot systems," in Proceedings of the 7th International Symposium on Experimental Robotics, pp. 10-13, Waikiki, Hawaii, USA, December 2000.

[39] W. J. Staszewski, K. Worden, R. Wardle, and G. R. Tomlinson, "Fail-safe sensor distributions for impact detection in composite materials," Smart Materials and Structures, vol. 9, no. 3, pp. 298-303, 2000.

[40] http://www.vallen.de/downloads. 


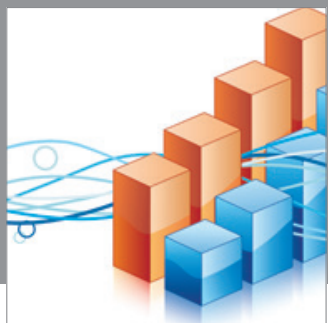

Advances in

Operations Research

mansans

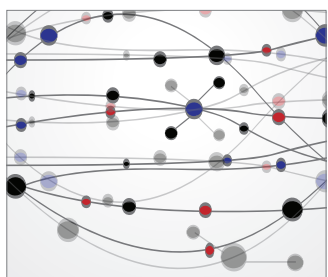

The Scientific World Journal
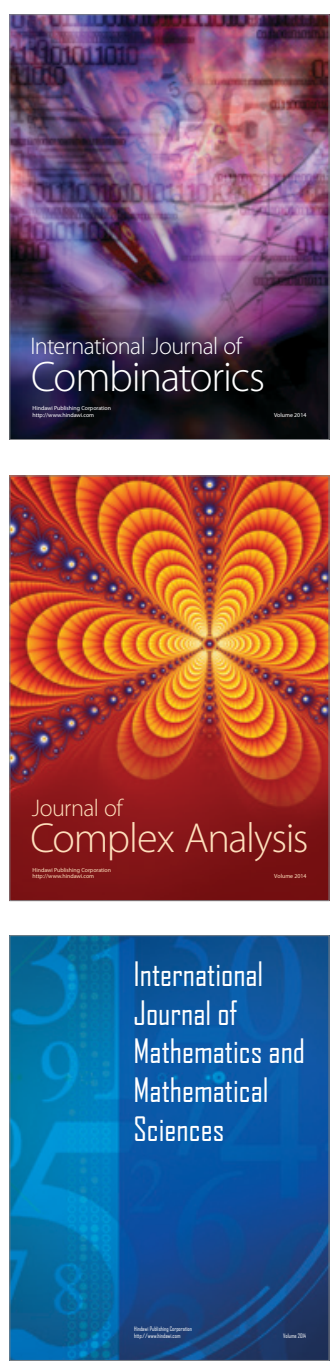
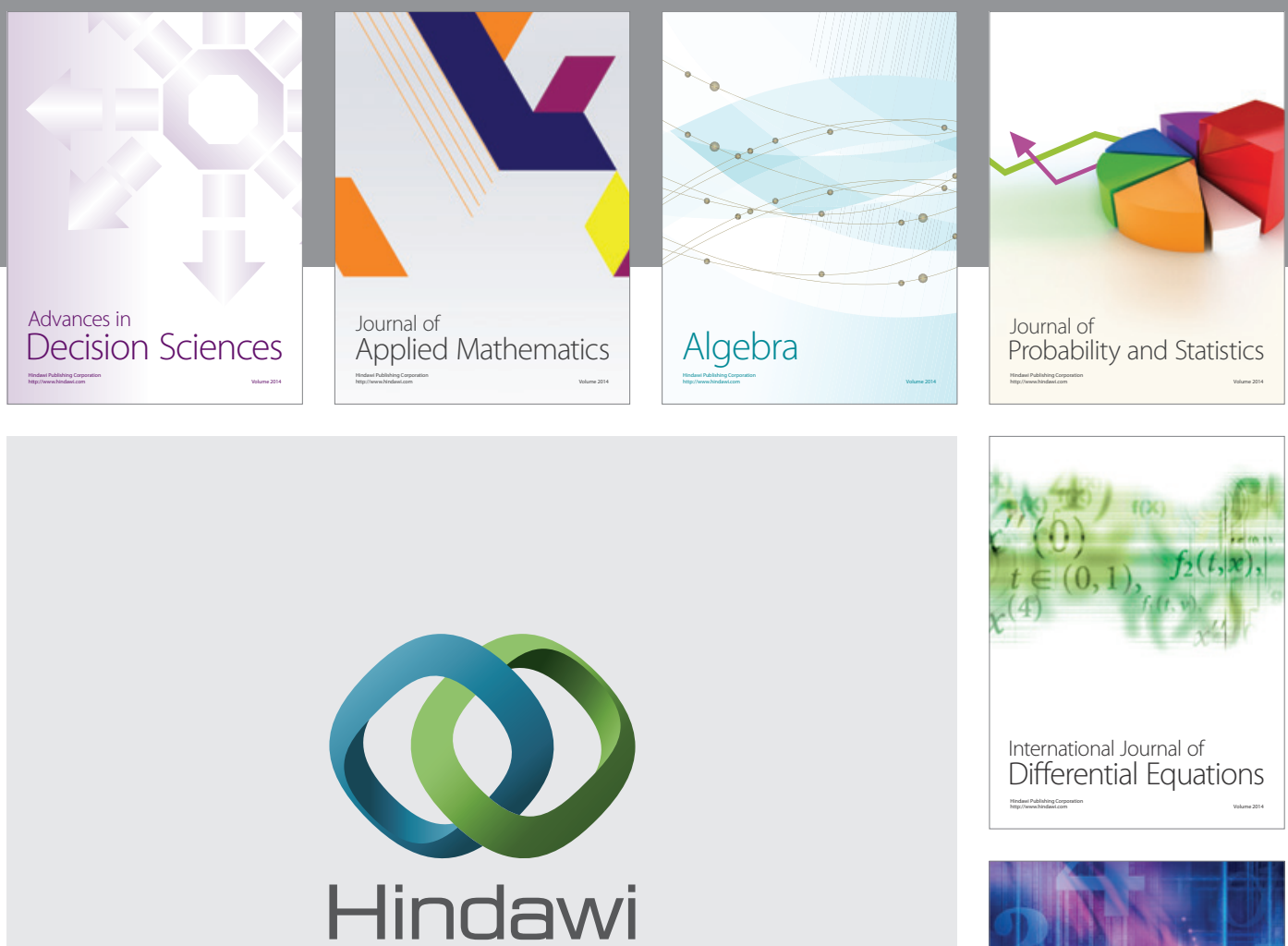

Submit your manuscripts at http://www.hindawi.com
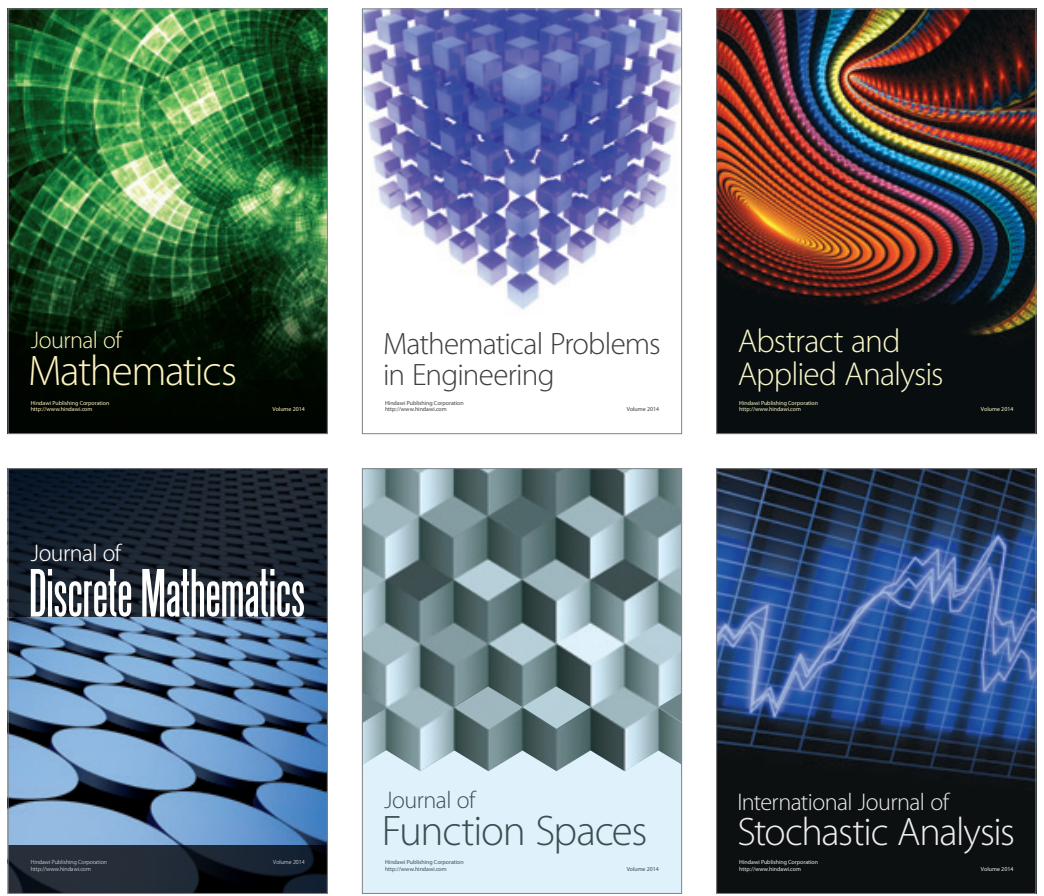

Journal of

Function Spaces

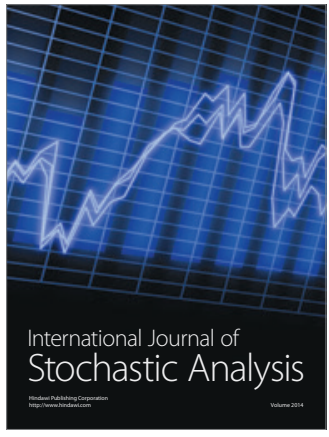

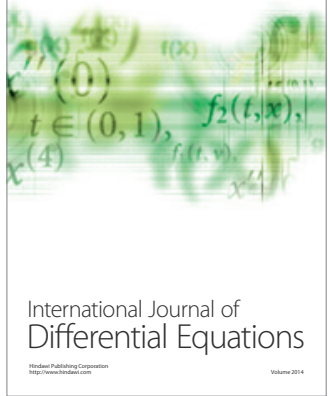
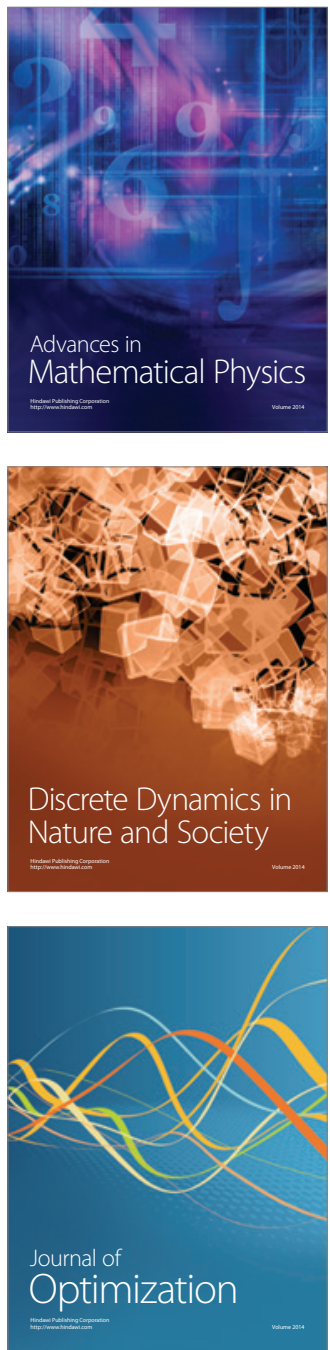